\title{
Exploration de l'activité de publication et de recherche de vidéos sur une plateforme audiovisuelle académique en ligne
}

Exploring the task of publishing and searching for videos on an online academic audiovisual platform

\section{Emmanuelle Papinot, André Tricot et Mônica Macedo-Rouet}

\section{OpenEdition}

Journals

Édition électronique

URL : http://journals.openedition.org/activites/3135

DOI : 10.4000/activites.3135

ISSN : $1765-2723$

Éditeur

ARPACT - Association Recherches et Pratiques sur les ACTivités

Référence électronique

Emmanuelle Papinot, André Tricot et Mônica Macedo-Rouet, « Exploration de l'activité de publication et de recherche de vidéos sur une plateforme audiovisuelle académique en ligne », Activités [En ligne], 15-1 | 2018, mis en ligne le 15 avril 2018, consulté le 22 avril 2019. URL : http:// journals.openedition.org/activites/3135; DOI : 10.4000/activites.3135

Ce document a été généré automatiquement le 22 avril 2019

\section{$(9) \Theta \Theta$}

Activités est mis à disposition selon les termes de la licence Creative Commons Attribution - Pas d'Utilisation Commerciale - Pas de Modification 4.0 International. 


\section{Exploration de l'activité de publication et de recherche de vidéos sur une plateforme audiovisuelle académique en ligne}

Exploring the task of publishing and searching for videos on an online academic audiovisual platform

Emmanuelle Papinot, André Tricot et Mônica Macedo-Rouet

\section{NOTE DE L'ÉDITEUR}

Article soumis le 30/05/2017, accepté le 06/11/2017

\section{Introduction}

1 Les activités de publication et de recherche de vidéo en ligne ont connu une croissance exponentielle au cours de cette dernière décennie. En à peine douze ans, le site YouTube est devenu un objet quotidien pour un milliard d'utilisateurs : chaque jour des centaines de millions d'heures de vidéos sont vues (Shearer, \& Gottfried, 2017). À ses débuts en 2005, les usagers publiaient surtout des contenus à des fins ludiques. Au fil du temps, le site de vidéos en ligne s'est diversifié, à tel point qu'il héberge aujourd'hui de nombreuses chaînes audiovisuelles destinées à des usages professionnels et universitaires. L'évolution des usages concerne également les normes et pratiques académiques, puisqu'on constate que les citations venant de vidéos publiées sur YouTube n'ont cessé de croître dans les publications scientifiques, principalement dans le domaine des Sciences Humaines et Sociales (Kousha, Thewall, \& Abdoli, 2012). En parallèle, des sites académiques spécialisés dans l'audiovisuel ont progressivement vu le jour, comme Canal-U - en 2001 en France qui propose par exemple une série de plus de dix heures consacrées à l'histoire de 
l'ergonomie. Si la fréquentation de ces sites est devenue une part importante de l'activité de nombreuses personnes, on sait encore peu qui sont ces usagers, la raison pour laquelle ces sites sont utilisés, en quoi une vidéo s'avère pertinente (i.e. à quels besoins informationnels elle répond), ni comment ces vidéos sont recherchées et trouvées. L'objectif de cet article est donc d'apporter un début de réponse à ces questions en interrogeant deux activités centrées sur la vidéo académique: la publication et la consultation.

\subsection{Usages et appréciation du média vidéo en contexte académique}

2 Un grand nombre d'études scientifiques sur l'usage du podcasts ${ }^{1}$ à des fins pédagogiques (Kay, 2012) montre l'intérêt croissant pour la vidéo dans les contextes académiques. Selon Kay, ces usages en contexte d'apprentissage varieraient considérablement en fonction des objectifs de l'usager, de la stratégie pédagogique employée ou encore de l'orientation scolaire. Ils concernent des conférences visionnées en classe ou en dehors, des vidéos venant compléter ou illustrer un cours, des vidéos produites par les apprenants euxmêmes (Heilesen, 2010) ou encore des vidéos proposant une démonstration (Ganier, \& De Vries, 2016) ou une résolution de problème. Heilesen attire l'attention sur le fait que les études longitudinales sur " les effets du podcasts » sont encore rares et qu'elles proposent la plupart du temps des résultats d'expériences effectuées sur un semestre ou deux après l'introduction de la vidéo en cours. L'introduction de la technologie pourrait donc légitimer l'enthousiasme constaté. Il ressort effectivement une "attitude affective positive » de la part des étudiants à l'égard de la vidéo qu'ils qualifient «d'agréable », «motivante » et qui «favoriserait l'attention» (Kay, 2012 ; Chen, \& Wu, 2015). D'autres facteurs expliqueraient cette satisfaction, notamment la créativité, l'accessibilité, l'indépendance qu'elle permet et l'augmentation de l'autoréflexion (Heilesen, 2010) ou encore une flexibilité dans les modes de visionnage (De Boers, Kommers, \& de Brock, 2011). Ces études rapportent des résultats disparates concernant l'amélioration de la performance scolaire, ainsi que certaines préoccupations, comme la capacité à s'auto discipliner, l'investissement d'un temps plus long qu'en présentiel ou encore le manque d'interaction et d'interactivité du média. Des raisons techniques justifieraient des difficultés d'usage, comme la taille des fichiers, le téléchargement, etc. Enfin, des facteurs cognitifs sont également évoqués, notamment une charge cognitive liée à la nature de l'engagement de l'intervenant, au ton de sa voix, au rythme du discours ou des conditions d'apprentissage peu maîtrisées par l'usager, par exemple lorsqu'il ne visionne que certains segments et perd ainsi la progression du discours et du raisonnement.

3 Si ces usages vont croissant, soutenus par les propositions technologiques, les nouvelles tendances éducatives ou encore les préférences des usagers qui recherchent de plus en plus la diversité et les avantages des nouveaux médias, les spécificités de la vidéo conduisent à se questionner sur les traitements nécessaires à sa mise en ligne en fonction des critères de pertinence pour la consultation.

\subsection{La vidéo : flux, traitements et pertinence}

4 La nature intrinsèque du média vidéo est décrite par Bachimont (1999) comme constituée de sons, d'images animées et d'une portée sémantique, intimement liés et difficilement dissociables. Il précise que sans traitement spécifique, la vidéo est peu aisée à consulter 
car son flux est linéaire: "le rythme de lecture d'une séquence est imposé par le document lui-même et non par le lecteur et le flux audiovisuel coïncide avec le flux de conscience ». Pour répondre à une indexation précise, deux niveaux doivent être pris en compte selon lui : la description locale d'une collection, d'une vidéo et de ses segments ( i.e. de chaque unité de contenu) et les relations entre les différentes unités décrites, afin de restituer la structure et l'organisation des ensembles et sous-ensembles. Ainsi, de façon optimale, la description et l'articulation de ces différents ensembles et unités peuvent parvenir à une relative autonomie, tout en apportant une cohérence dans la relation entre chaque niveau de sens, inscrit dans le tout.

5 Afin de rechercher des critères de pertinence propres à la vidéo, basés sur le besoin des usagers, Yang et Marchionini (2004) ont mené une étude exploratoire auprès de quatre experts (deux enseignants, un bibliothécaire spécialisé dans l'audiovisuel et un éditeur de vidéos). Les auteurs ont mis en avant les caractéristiques spatio-temporelles de la vidéo, l'hétérogénéité des usagers, la diversité de leurs besoins ainsi que la complexité à "décoder le contenu d'une vidéo ». Leurs résultats aboutissent à trois catégories de critères. La première, «textuelle», repose principalement sur le topic, l'actualité et la provenance, alors que la deuxième, "visuelle ", correspond à des informations sur la cinématographie, les objets, les évènements ou le style. La troisième catégorie regroupe des informations plus subjectives, liées aux expériences de l'usager et aux tâches (critères d'intérêt, de familiarité et de pertinence). Ils ont ainsi conclu que les critères de pertinence sont beaucoup plus complexes pour la vidéo que pour les documents textuels.

6 La mise à disposition de vidéos en ligne amène à se questionner sur l'interaction entre l'environnement qui diffuse les vidéos et les modes de recherche que les usagers mettent en place pour les trouver.

\subsection{Recherche d'information, dispositifs audiovisuels et vidéos}

7 Concernant la recherche sur la vidéo et le développement de dispositifs audiovisuels académiques en ligne, une communauté semble incontournable dans le paysage international. Le National Institute of standards and Technology (NIST) et l'Advanced Research and Development Activity (ARDA) qui avaient préalablement lancé le Text REtrieval Conference (TREC ${ }^{2}$ ) en 1992, créent en 2001, le TREC Video Retrieval Evaluation (TRECVid) ${ }^{3}$. La Communauté scientifique du TRECVid répond à des lignes directrices qui lui sont fournies annuellement. Les consignes dispensées en 2017 mettaient en avant les thèmes suivants : "Video Search", "Surveillance event detection", "Instance search", "Multimedia event detection", "Video to Text» et "Video Hyperlinking»". Au moment de la création du TRECVid, afin de proposer un terrain d'études à cette communauté, Gary Marchionini met en place l'open Video Digital Library (Marchionini, Wildemuth, \& Geisler, 2006) qui rassemble des collections audiovisuelles destinées à la recherche et à l'enseignement supérieur, accessibles au grand public. Des études empiriques qualitatives et quantitatives sur les usages réels, menées aussi bien sur les processus semi-automatisés que sur les pratiques manuelles ont été intensément effectuées jusque vers 2010 puis, peu à peu, les consignes se sont recentrées sur les métriques et la recherche automatisée de vidéos. Ces études apportent une distinction entre la recherche de vidéo dans un dispositif (video browsing), i.e. la navigation dont le but est d'atteindre une ressource ou une information et l'évaluation du contenu d'une vidéo (video retrieval), i.e. la compréhension de la structure du contenu et de ses informations (Marchionini, Song, \& Farrell, 2009). 
Certaines expérimentations sur le video browsing se sont intéressées aux requêtes dynamiques, à la qualité des feedbacks ou encore au contrôle-utilisateur à travers la flexibilité des interfaces (AgileViews) (Marchionini, Geisler, \& Brunk, 2000). Ces études ont testé des interfaces interactives qui prenaient en compte la relation entre les niveaux de structure du fonds, la granularité des informations et leurs différentes natures. Des mécanismes de contrôle étaient manipulés par l'usager qui pouvait afficher différents niveaux de représentation en fonction de ses préférences et de ses besoins. Il pouvait par exemple combiner des vues d'ensemble des collections audiovisuelles avec des prévisualisations des détails des collections et des vidéos (Greene, Marchionini, Plaisant, \& Shneiderman, 2000). À partir de la fin des années 1990, un certain nombre d'expérimentations sur le video retrieval s'est centré sur ce que Marchionini nomme « les substituts » (surrogates), des aides permettant d'obtenir rapidement une représentation compacte du contenu d'une vidéo, avec ou sans requête explicite. Trois types de substituts ont été expérimentés : les substituts audio, textuels et visuels, comprenant des images fixes ou en mouvement (Wildemuth, Marchionini, Wilkens, Yang, Geisler, Fowler, \& Mu, 2002; Song, \& Marchionini, 2007). Les résultats ont tout d'abord montré la prédominance des substituts textuels dans la décision de visionnage et l'appréciation d'un substitut visuel pour conforter ce choix (Ding, Marchionini, \& Soergel, 1999; Hughes, Wilkens, Wildemuth, \& Marchionini, 2003). Ils ont également montré que l'audio seul était plus utile que le visuel seul, tout en concluant que la combinaison de plusieurs substituts l'emportait systématiquement, surtout lorsqu'ils étaient produits manuellement et qu'ils étaient pertinents par rapport à la nature du contenu de la vidéo et à son style (Song, \& Marchionini, 2007). Ayant étudié la relation entre le choix de substituts et les caractéristiques du contenu, les exigences de la tâche et les préférences des usagers (Wildemuth, \& al., 2002 ; Yang, \& al., 2003), les chercheurs ont conclu qu'on ne peut généraliser les besoins et les préférences des utilisateurs. La revue proposée par Schoeffmann (2010) rappelle les travaux de Marchionini et la difficulté à produire un substitut pertinent et efficace, adéquat au contenu de la vidéo mais souligne également que le domaine concerné par le corpus audiovisuel doit déterminer la technique d'analyse du contenu, le besoin de segmentation et sa granularité. Des travaux plus récents s'intéressent au marquage du flux pour favoriser la navigation mais également à l'aide apportée par un index et une table des matières interactifs (Merkt, \& Schwan, 2014; Cojean, \& Jamet, 2017).

8 Si les travaux que nous venons de citer permettent de mieux comprendre les enjeux et la complexité de la recherche de vidéo en ligne, on peut cependant constater qu'ils sont axés sur les extrémités des phases du parcours de recherche, i.e. l'appréhension de la présentation du fonds audiovisuel d'un côté et l'évaluation du contenu d'une vidéo et de la navigation dans le flux de l'autre. Contrairement à la recherche d'information "traditionnelle", il n'existe à notre connaissance aucun modèle d'analyse complet, spécifique à la recherche de vidéo. C'est la raison pour laquelle nous présentons maintenant un modèle général de recherche d'information. Parmi les différents modèles disponibles, centrés sur l'activité située, la théorie de l'Information foraging (Pirolli, \& Card, 1999) et les différents modèles qui en découlent se distinguent par leur amplitude et l'adaptabilité qu'ils proposent. Le comportement complexe de l'usager est analysé à travers l'explicitation de ses interactions avec l'environnement qui participent à la construction du sens de son parcours de recherche (Pirolli, 2007). Le modèle cognitif de Pirolli (2007), ACT-SCENT Architecture, s'apparente à un modèle de mémoire qui fait 
interagir la définition du but par l'usager, l'activation de l'information et l'action issue de la cognition dans la résolution du problème. L'Information foraging place la notion d'utilité au cœur de la recherche, en justifiant l'engagement d'un individu dans celle-ci par une estimation d'un gain supérieur au coût. À partir de là, l'individu poursuit deux buts : le but informationnel (qui peut être satisfait par l'information ou la ressource trouvée mais également par l'acquisition de connaissances liée à l'action en cours) et celui de l'activité principale qui motive la recherche (i.e. une activité dont on ne sait pas comment atteindre le but mais dont la représentation de la solution correspond à une vidéo). Tout au long de son parcours, l'usager est amené à « modeler l'environnement $»^{5}$ en effectuant une navigation entre des grappes d'informations basées sur le choix de signaux proches (accès, fonctionnalités, services spécifiques, etc.), qui lui permettent de sélectionner les zones qui s'avèrent utiles pour se rapprocher de son but. Dans le même temps, il doit suivre une information intermédiaire, le "parfum " ${ }^{6}$, qui lui permet d'appréhender au fur et à mesure la pertinence de la ressource disponible. Le cadre qu'offre la théorie de l' Information foraging et ses modèles adaptatifs très développés permet d'intégrer les résultats d'études scientifiques sur la recherche d'informations spécifiques à l'audiovisuel, visant la production d'un cadre à la fois pratique et théorique (Marchionini, Wildemuth, \& Geisler, 2006). Dans cette étude, le modèle appliqué à la recherche de vidéo a déterminé un périmètre qui s'étend depuis la décision de prise en charge de la recherche jusqu'à la décision de visionnage d'une vidéo, décision qui peut reposer sur une évaluation du contenu de celle-ci, à travers l'usage de substituts ou en effectuant un visionnage partiel de la vidéo présélectionnée. Si l'usage de la vidéo ne fait pas l'objet de cette étude (i.e. ce que l'usager fait de cette vidéo ou des informations qu'elle contient, une fois qu'il l'a sélectionnée), la déclaration des conditions de visionnage a priori, i.e. l'intention de visionner la vidéo sélectionnée de telle ou telle façon, fait en revanche partie intégrante des éléments déterminant la recherche.

\subsection{Analyse de l'activité centrée sur la vidéo académique en ligne}

9 Cette revue de littérature a montré d'une part, qu'il existe une activité importante basée sur l'utilisation croissante de vidéos académiques qui s'accompagne d'une évolution des usages de ces vidéos dans des contextes professionnels, et d'autre part, que l'activité de recherche de vidéos académiques est encore sous-étudiée dans la recherche, du point de vue ergonomique pour le moins.

10 Deux études complémentaires sur la publication et la consultation ont été menées afin de mieux comprendre les enjeux de l'activité centrée sur la vidéo académique en ligne. L'objectif de la première étude cherche à évaluer dans quelle mesure la réalisation de la tâche de publication peut impacter l'activité de consultation. La seconde étude présente un profil fonctionnel de l'usager à travers sa représentation mentale de l'activité de recherche (video browsing) et de consultation de vidéos (video retrieval). Il s'agit de comprendre les raisons pour lesquelles un sujet choisit d'utiliser tel système pour rechercher de l'information, ce qu'il cherche (Tricot, \& Nanard, 1998) et comment il cherche.

11 Ces études exploratoires ont été effectuées sur le dispositif Canal-U, une grande banque d'archives audiovisuelles, dédiée à l'Enseignement Supérieur et à la Recherche. En 2016, le dispositif propose une production audiovisuelle de 12261 heures de vidéos diffusée en ligne, en accès libre (4911000 visites estimées $\left.{ }^{7}\right)$, composée en grande partie de 
conférences et d'interviews en streaming. Cette production repose sur un réseau de 160 " contributeurs $»^{8}$, correspondant principalement à des services institutionnels qui gèrent la chaîne en ligne qu'ils ont créée sur le dispositif, dont ils sont responsables et sur laquelle ils publient leurs vidéos.

La description de la tâche inclut différents aspects, principalement le but à atteindre, l'environnement (et ses outils) et le mode opératoire (i.e. l'action menée pour atteindre le but) (Hoc, \& Leplat, 1983). Lorsque l'usager débute une recherche sans savoir comment atteindre son but, la recherche d'information devient l'activité principale et s'inscrit dans une situation de résolution de problème dans laquelle la relation entre l'usager et la tâche devient centrale. L'activité est décrite à travers la hiérarchie de la tâche, pour la publication et la consultation, les facteurs qui conditionnent sa réalisation ainsi que les spécificités issues des différents types de buts et modes opératoires rencontrés. L'exposition des résultats pour chaque étude comporte deux parties: tout d'abord les résultats liés à la tâche effective commune, i.e. ce qui est réalisé de façon commune par l'ensemble des usagers puis ceux présentant la diversité des modes opératoires rencontrés.

\section{Méthodologie de l'étude 1}

Cette étude s'est effectuée en deux étapes. Tout d'abord, une inspection heuristique (Bastien, Leulier, \& Scapin, 1998) (Annexe 1 - Graphe 1) a servi de base à la création de fiches de description permettant de produire un graphe de la tâche a priori, i.e. ce qui doit être fait par l'opérateur pour atteindre son but (Annexe 1 - Graphe 2). La visualisation de la tâche a priori devait fournir une référence pour l'observation de la tâche effective, i.e. ce qui est réellement fait par l'opérateur.

Dans un deuxième temps, des entretiens semi-dirigés par saturation ont été conduits auprès de sept opérateurs, responsables de la publication de vidéos sur leurs chaînes audiovisuelles, en ligne sur le dispositif étudié. La sélection de cet échantillon reposait sur l'hétérogénéité des institutions ( 4 universités, 2 centres de recherche et une institution privée reconnue d'utilité publique), des organisations internes des services de publication audiovisuelle (un opérateur seul ou en équipe) ainsi que des différents métiers et statuts des opérateurs. Les professionnels interviewés pratiquent cette activité de publication audiovisuelle depuis plus de 10 ans (sauf un ingénieur pédagogique et un responsable de service audiovisuel). Le type de publication audiovisuelle étudiée relève donc d'un cadre maîtrisé et contrôlé par une activité professionnelle. Aucun opérateur n'a déclaré avoir reçu de formation en indexation audiovisuelle au cours de sa carrière, cependant deux opérateurs sont des documentalistes et à ce titre, ils ont été formés aux techniques d'indexation des documents écrits. Les autres participants ont des statuts divers: technicien et réalisateur audiovisuels, ingénieur en technologie de l'information, responsable de service audiovisuel et chef de projet web. Des entretiens sur le poste, d'une durée de $2 \mathrm{~h}$ à $3 \mathrm{~h}$ chacun, ont été conduits sur le lieu de travail des interviewés, avec leur propre matériel. Un enregistrement audio a systématiquement été effectué avec l'accord des participants. Deux techniques de conduite d'entretien ont été employées : la technique du «Pourquoi? Comment?» (TPC) servant à collecter les connaissances de l'opérateur sur la planification d'une série d'actions qu'il réalise habituellement (Sébillotte, 1991; Bisseret, Sébillotte, \& Falzon, 1999) et la technique des protocoles verbaux (TPV) visant à collecter des données concernant la description de la procédure 
telle qu'elle se déroule réellement. Cette deuxième technique a été utilisée ponctuellement, par exemple lorsque le sujet n'arrivait pas décrire la tâche ou lorsqu'il déclarait détourner les fonctionnalités initiales de l'outil de publication pour satisfaire son but. Le guide d'entretien était divisé en six chapitres (1. Statut, rôle et missions du responsable de publication; 2. Description de chaque tâche couramment effectuée; 3. Critères d'indexation des données audiovisuelles et périmètre de l'expertise éditoriale ; 4. Conception et présentation de la chaîne audiovisuelle en ligne; 5. Place de Canal-U dans l'activité du contributeur ; 6. Satisfaction par rapport au dispositif et aux outils).

Les entretiens ont été transcrits dans leur totalité et analysés grâce à la Méthode Analytique de Description (Sébillotte, 1994) qui permet de restituer les tâches de façon hiérarchique, en tenant compte à la fois des objectifs visés par les opérateurs mais aussi de la façon dont ils les atteignent et des contraintes et des difficultés qu'ils rencontrent. Il s'agit de repérer la description de chaque tâche et sous-tâche évoquées dans le discours de l'usager puis de les relier entre elles selon leurs relations hiérarchiques ${ }^{9}$ de façon à obtenir une description la plus complète possible de l'ensemble des tâches réalisées par l'usager pour atteindre son but. Dans cette description hiérarchique, sont également consignés les attributs de la tâche, ses conditions d'exécution, son état initial et final ainsi que des évènements qui peuvent survenir au cours de sa réalisation. Cette formalisation a permis d'une part, de construire une représentation mentale de l'activité de l'usager en apportant des précisions sur sa situation, la perception de son but et de la réalisation de la tâche, et d'autre part, de catégoriser les procédures mises en œuvre d'après les répondants (Bisseret, Sébillotte, \& Falzon, 1999).

\section{Résultats de l'étude 1 : impacts de l'activité de publication audiovisuelle sur la tâche de consultation}

16 L'activité de publication concerne la réalisation de trois types de tâches. Le premier correspond à la manipulation de "l'objet-vidéo » et se traduit par des tâches d'encodage qui se finalisent par l'upload d'un ou plusieurs fichiers dans l'outil de publication. Le deuxième repose sur la production d'informations sur et autour de la vidéo, i.e. une tâche d'indexation qui aboutira au minimum à la création d'une notice accompagnant la vidéo en ligne. Le troisième type de tâches vise à effectuer un classement multiforme qui participe à la transformation de la vidéo en archive. Il repose sur une analyse qui se manifeste à travers la production de repères intellectuels (par exemple l'inscription dans une classification normée comme Dewey) et de codes (par exemple l'ajout de tags à l'aide de mots-clés). Il consiste également à localiser la vidéo dans une ou plusieurs organisations préexistantes (par exemple dans un classement thématique ou une collection). Ce troisième type de tâches a obligatoirement pour prérequis l'existence des deux précédentes et comprend un aspect réflexif sur l'ensemble des vidéos existantes et à venir, dans le fonds et les collections.

\subsection{La tâche effective commune de publication}

17 La tâche effective commune à l'ensemble des responsables de la publication, i.e. la tâche minimale effectuée, correspond aux tâches rendues obligatoires dans l'outil de publication, à l'exception de la tâche de classement d'une vidéo dans une collection, initialement facultative et pourtant systématiquement réalisée (Annexe 2 - graphe 7). Un 
premier type de contraintes est signalé par l'ensemble des opérateurs. Il s'agit d'un point de rupture qui peut survenir dans plusieurs situations et qui aboutit à la "destruction " du travail entamé, à travers la suppression de la vidéo publiée ou en attente de publication. Lorsqu'elle se produit au tout début de la chaîne de publication, elle s'apparente à une contrainte relationnelle puisqu'elle vient d'une absence de transmission de la documentation ou de l'autorisation de diffusion qui doit accompagner la captation. Les responsables de la publication signalent également une contrainte de droits d'auteur lorsque la destruction de la vidéo est effectuée à la demande d'un intervenant qui ne souhaite plus que son intervention soit diffusée. Cette contrainte devient d'ordre technique lorsqu'on doit supprimer la vidéo suite à un problème de format qui rend le média illisible (par exemple lors d'une migration d'un format à un autre). Un deuxième type de contraintes peut apparaitre tout au long de la chaîne de publication lorsque l'absence d'information ou de guidage ne permet pas à l'opérateur de comprendre les enjeux de la tâche à réaliser (par exemple une explicitation des différents formats d'encodage). Cette contrainte cognitive peut se transformer en un jeu de contraintes circonstancielles si l'opérateur suit une procédure inappropriée ou complexifie sa tâche (Chevalier, \& Cegarra, 2008).

On constate enfin que les tâches facultatives effectuées traduisent la diversité des modes opératoires. On les retrouve principalement dans le degré de réalisation des tâches d'indexation et de classement, puis dans les tâches de post production (par exemple dans la mise à disposition de documents accompagnant la vidéo ou la proposition d'une segmentation de la vidéo ${ }^{10}$.

19 La classification des différents modes opératoires a permis d'observer deux types de pratiques marquées, associés à deux types de profils responsables de la publication: le «non-documentaliste » et le documentaliste.

\section{La tâche de publication perçue par les non-documentalistes}

20 La catégorie des non-documentalistes regroupe des statuts variés dont les compétences principales résident pour la majorité dans un périmètre d'intervention qui s'exprime avant la publication, i.e. dans la réalisation et le montage de la vidéo. Certains effectuent la mise en ligne par défaut et la majorité déclare avoir pour objectif de procéder à une indexation «la plus rapide possible» qui leur prend de « 5 à 10 minutes» (Annexe 2 graphe 5). La tâche de publication s'apparente à une routine pour eux (Rasmussen, 1983) et repose sur des procédures dont le contrôle leur échappe plus ou moins, qu'ils avouent avoir du mal à verbaliser et dont le résultat peut être incertain : "En fait comme il y a les onglets, je fais les onglets les uns après les autres» (Annexe 2 - graphe 6, opérateur A). Leur objectif engendre un comportement réactif qui cherche des procédés pour " réussir à publier» (Leplat, 2006) et peut s'inscrire dans ce que Falzon (1994) nomme des activités fonctionnelles orientées vers la production immédiate. La plupart déclare effectuer parfois quelques tâches optionnelles, comme l'ajout de documents téléchargeables lorsqu'on les leur fournit ou la segmentation de la vidéo pour "faciliter le visionnage pour le visiteur" (Annexe 2 - graphe 3). Il s'agit pour eux de découper rapidement les moments principaux de la vidéo :

Comme sur le programme j'ai à peu près les intervenants, je les retrouve sur la vidéo et je tope le temps, c'est toujours des gens qui parlent une vingtaine de minutes. Après chaque intervenant, on fait une coupure. (Annexe 2 - graphe 3). 
21 Ils souhaiteraient donc un outil «plus automatisé » (Annexe 2 - graphes 3, 5 et 6 opérateur A) qui leur permettrait de gagner du temps. Des évènements non souhaités (Leplat, 2006) verbalisés dans les tâches de classement permettent de relever certaines difficultés qui pourraient expliquer des «zones d'incertitude» (Crozier, 1977) dans l'ensemble du dispositif. La plupart signale par exemple une difficulté à évaluer la performance de leur tâche :

Je ne fais pas partie des circuits des documentalistes, je ne suis pas reconnu comme quelqu'un pouvant produire une norme. Ce que je mets comme mots-clés, est-ce que ça suffit? Est-ce que ça ne sert à rien? Je ne sais pas » (Annexe 2 - graphe 5). D'autres soulignent une absence de conception : « Il n'y a pas vraiment de réflexion, chaque titre de séminaire devient une collection quasiment. C'est une espèce d'organisation au fil de l'eau dont la validation se fait par les gens qui ont produit le contenu. (Annexe 2 - graphe 3).

Ce profil se déclare globalement satisfait de l'utilisabilité de l'outil de publication, ce qui s'explique en grande partie par une longue pratique professionnelle qui a suivi les évolutions technologiques de la publication audiovisuelle et une activité basée sur l'appropriation d'un outil utilisé au quotidien. L'utilité se confondant facilement avec l'utilisabilité - l'objectif étant uniquement la réalisation de la tâche pour finaliser la publication, sans réflexion sur l'activité, l'usage final ou l'amélioration documentaire - on est face à un objectif fermé sur l'outil lui-même. La performance est associée au souhait d'une plus grande automatisation qui pourrait alléger la tâche.

\section{La tâche de publication perçue par les documentalistes}

Les documentalistes revendiquent des compétences-métier et une pratique délibérée (Tricot, 2005). Ils déclarent effectuer une indexation "la plus complète et précise possible pour fiabiliser l'information» (Annexe 2 - graphe 4), dans laquelle ils incluent les tâches de classement documentaire :

J'essaie d'extraire la grande discipline dans le classement thématique, après je complète par la Dewey et ensuite j'affine par les mots-clés. La triple indexation c'est important, parce chaque indexation ne va pas forcément se recouper. Elles vont essayer d'aller de plus en plus finement vers l'objet que je suis en train d'indexer. (Annexe 2 - graphe 6, opérateur B).

Ils estiment la réalisation de la tâche à plus d'une heure de travail pour chaque vidéo, audelà de son visionnage préalable complet. La plupart du temps, ils la visionnent en entier en prenant des notes avant de débuter la production d'informations et les traitements annexes, sans perdre de vue la finalité de la tâche:

Une segmentation de la vidéo ne peut être en lien qu'avec le contenu, ce n'est pas une forme! Si c'est pour dire l'intro et la conclusion, je pense que ce n'est pas le niveau de notre public! (Annexe 2 - graphe 4).

La difficulté pour eux ne réside pas dans la réalisation de la tâche proposée mais dans le détournement des possibilités trop restrictives du système (par exemple un classement thématique qui ne convient pas à la complexité et à la diversité du contenu). Ils opèrent alors une régulation par la procédure (Leplat, 2006). L'un des documentalistes interrogés (Annexe 2 - graphe 4) précise également que l'indexation qu'il effectue actuellement n'est en rien spécifique à la vidéo car le dispositif ne le permet pas. La description qu'il fait de l'indexation audiovisuelle souhaitée rejoint les « critères visuels » mis au jour par Yang et Marchionini (2004), comprenant la précision des techniques cinématographiques, la description des objets, personnes et évènement diégétiques, etc. 
26 L'objectif des documentalistes repose sur la performance de la tâche qui peut générer de nouvelles contraintes liées aux actions alternatives qu'ils mettent en place. Leur activité peut être qualifiée de méta fonctionnelle (Falzon, 1994), i.e. orientée vers la « construction de connaissances et d'outils destinés à une réutilisation ultérieure ». Cette réflexion professionnelle les amène par exemple à s'impliquer dans le choix d'un système documentaire qui répondrait à la complexité de leur travail tout en prenant en compte la consultation par un public non expert :

Il existe des formats de description MARC pour la vidéo, ou le MPG7 en format de description vidéo, mais c'est tellement complexe que personne ne l'utilise. [...] RAMEAU de la Bibliothèque nationale ou du SUDOC, ce n'est pas gérable [...] Il faut atteindre un équilibre entre la documentation scientifique, la visibilité et l'accessibilité surtout grand public, parce que la vidéo pour moi, c'est un outil grand public. C'est cet équilibre-là qui n'est pas évident à maintenir. (Annexe 2 - graphe 6 , opérateur B).

27 Leur réflexion concerne également le respect des normes et des bonnes pratiques ainsi qu'un guidage (par exemple via des thesauri, un vocabulaire contrôlé, etc.) qui permettrait d'assurer la cohérence générale du système documentaire. L'organisation des collections et leur présentation pour le visiteur demeure une question ouverte. Leur choix se porte par défaut sur « une arborescence qui reprend celle des centres institutionnels qui facilite l'administration des collections", mais "qui n'est ni attractive pour le visiteur ni signifiante ». Cette solution s'avère cependant selon eux plus satisfaisante que «la typologie d'évènements peu interactive pour l'usager, qu'un classement par discipline qui ne convient pas aux institutions interdisciplinaires ou qu'une présentation par mots-objets» (Annexe 2 graphe 6, opérateur B).

28 La préoccupation majeure des documentalistes concerne l'utilité de leur travail dans l'environnement proposé et se rapproche de la notion d'acceptabilité puisque la réflexion documentaire et l'amélioration éditoriale de la publication audiovisuelle sont d'après eux, partie prenante de leur métier. La performance pour eux n'est pas bornée et leur souhait d'amélioration vise une extension des possibilités éditoriales de l'outil de publication pour mieux répondre à l'évolution de leurs besoins.

\subsection{Conclusion de l'étude 1}

Cette étude exploratoire sur l'activité de publication a révélé que les compétences des responsables de la publication ne portent pas sur la même partie de la chaîne. Pour les uns, elles relèvent principalement de la production dont la publication représente la finalité de leur activité. Pour les autres, elles s'appliquent à l'indexation et au classement documentaire qui sont au cœur de leur métier. Les difficultés que rencontrent les différents responsables de la publication ne sont pas localisées sur les mêmes tâches à l'exception des tâches d'encodage dont la finalité - en lien avec la qualité de la connexion internet du visiteur qui conditionne le visionnage de la vidéo - n'est pas toujours comprise. La tâche d'indexation s'avère double. La tâche minimale doit être réalisée par l'ensemble des responsables de la publication afin de garantir la mise à disposition d'une notice complète, comprenant des informations exactes produites dans le respect des normes intelligibles pour le visiteur. La tâche d'indexation complexe concerne des tâches facultatives et repose sur le degré de réalisation de la tâche, conditionnée à la fois par les compétences de l'individu mais également par les possibilités de l'outil. Enfin, une réflexion est portée sur les tâches de classement prévues pour assurer la cohérence 
générale du dispositif, son interopérabilité mais également l'identité des chaînes audiovisuelles à travers leurs collections. Trois types de difficultés sont observés : celles qui dépendent de la conception du système et notamment de la classification d'une ressource dans un ensemble préexistant (par exemple la définition des thématiques du fonds), celles qui relèvent de la responsabilité des chaînes audiovisuelles (principalement la conception des collections) et enfin celles qui reposent sur les compétences individuelles (par exemple la compréhension d'une classification comme Dewey).

\section{Méthodologie de l'étude 2}

méthode décrite pour l'étude 1 , à savoir une inspection heuristique de la plateforme en ligne Canal-U (Bastien, Leulier, \& Scapin, 1998) à partir des scénarios de consultation de vidéos. Cette inspection a servi de base à la création de fiches de description (Sébillotte, 1994) permettant de produire un graphe de la tâche a priori, i.e. ce qui doit être fait par l'usager pour atteindre son but (Annexe 4 - graphe 8).

31 Huit usagers ont été interrogés après avoir été recrutés via leur compte personnel de stockage ouvert sur le dispositif audiovisuel étudié et un appel à participation auprès des réseaux sociaux professionnels et de l'université Paris 8 . Il s'agissait à nouveau d'un échantillon de convenance (Robinson, 2014) basé sur des critères d'hétérogénéité et d'accessibilité, qui a retenu deux étudiants de master à distance, un enseignant de français langues étrangères, trois ingénieurs pédagogiques d'université, un enseignantchercheur et un usager sans emploi présentant un profil hors Enseignement Supérieur et Recherche. Six usagers sont français et résident en France et les deux autres sont étrangers et résident en dehors ${ }^{11}$. Des entretiens semi-dirigés par saturation ont été conduits en direct pour cinq d'entre eux et via un outil de visioconférence ${ }^{12}$ pour les trois autres. La première partie du guide d'entretien reposait sur la description des tâches couramment effectuées et l'explicitation de la réalisation de la tâche (Sébillotte, 1991). La seconde partie reposait sur trois variables, la première visant à approfondir la description du contexte ou de la situation de l'usager au moment de la prise en charge de la recherche, les deux autres, à mieux comprendre les résultats de l'action issue de la cognition, à travers deux aspects qualitatifs du modèle de l'Information foraging (Pirolli, \& Card, 1999) : le modelage de l'environnement (le choix d'accès et de signaux proches) et le parfum (l'activation de l'information intermédiaire). Enfin, afin de répondre aux spécificités de la vidéo, les résultats des études sur l'évaluation du contenu audiovisuel (Marchionini, Song, \& Farrell, 2009) ont été transformés en question et combinés avec celles issues des dimensions précédemment citées (besoin d'information, modelage de l'environnement et parfum). Chaque entretien a duré environ deux heures, enregistré en audio et transcrit dans son ensemble.

Une première analyse s'est centrée sur la tâche et sa formalisation MAD (Sébillotte, 1994; Bisseret, Sébillotte, \& Falzon, 1999). Une deuxième analyse a été effectuée à partir des variables permettant la description du but de l'usager et de sa situation au moment de la prise en charge de sa recherche (Tricot, 2015), son interaction avec l'environnement notamment à travers les signaux et les informations qui influent sur sa stratégie (Pirolli, \& Card, 1999), ainsi que son comportement dans les phases de l'activité spécifiques au média vidéo (par exemple Marchionini, Song, \& Farell, 2009). 


\section{Résultats de l'étude 2 : l'activité de consultation de vidéos}

Les résultats ont produit un découpage de l'activité en quatre phases, correspondant à un parcours en profondeur dont les différents niveaux abritent quatre types de grappes d'informations : le fonds, les collections, les données et métadonnées d'une vidéo et le contenu de la vidéo. Lors de la première phase de l'activité, l'usager cherche à appréhender l'organisation du fonds global afin de choisir un accès sur la page d'accueil. Dans la deuxième phase de son activité, il devra procéder à un parcours intermédiaire (sur différentes interfaces) qui le conduira à présélectionner une vidéo. La troisième phase consiste à évaluer la notice de la vidéo, d'une part en confirmant la pertinence des informations déjà évaluées au niveau supérieur et d'autre part, en recherchant de nouvelles informations pour conforter la sélection. Enfin la quatrième phase correspond à l'évaluation du contenu de la vidéo.

\subsection{La tâche effective commune de consultation}

La tâche effective commune restitue l'analyse des données communes à l'ensemble des usagers interrogés.

\section{Description de la tâche effective}

Dans l'environnement étudié, lors de la première phase de l'activité, l'usager doit choisir un accès sur la page d'accueil (Annexe 3 - figure 1) sans connaître ni la logique de classement utilisée, ni le périmètre du fonds disponible. La tâche effective commune à l'ensemble des visiteurs se résume à l'utilisation d'un seul accès sur la page d'accueil : le moteur de recherche (Annexe 4 - graphe 9). Les usagers interrogés déclarent que cet accès est devenu un standard pour l'ensemble des sites qu'ils consultent, on constate cependant un usage par défaut qui se justifie par une densité d'informations dissuasives sur la page d'accueil, une incompréhension de la présentation des collections (Annexe 3 figure 3) ou par un accès thématique qui génère trop d'efforts. Ne pouvant contextualiser la ressource recherchée, l'usager préfère alors redonner une partie du contrôle au système (Annexe 3 - figure 2). Dans la troisième phase, la tâche commune d'évaluation des informations de la notice (Annexe 3 - figure 4) repose principalement sur une confirmation du titre. Les stratégies se divisent ensuite: l'un des usagers ne lit pas la notice au-delà du «sujet» alors que d'autres évaluent un nombre d'informations importantes, parfois de façon exhaustive. À ce stade, de nombreuses difficultés signalées sont provoquées par une indexation peu précise (un titre différent sur la notice et sur la vidéo) ou erronée (une imagette peu représentative du contenu qui induit en erreur). Un grand nombre de tâches alternatives apparaissent dans la phase quatre de l'activité. Audelà des tâches traditionnelles d'évaluation du contenu avant le visionnage, i.e. la lecture du résumé ou des mots-clés ${ }^{13}$, les usagers usent de différents moyens pour comprendre la structure du contenu de la vidéo (en lisant par exemple les documents d'accompagnement) ou pour obtenir une idée de l'orientation du discours (en écoutant par exemple les premières minutes de la vidéo). Etant donné que certains usagers déclarent sélectionner une vidéo dans la liste de résultats du moteur de recherche, sans évaluation de la notice ni du contenu avant le visionnage, la tâche effective commune à 
l'ensemble des usagers se réduit à l'usage du moteur de recherche sur la page d'accueil et à l'évaluation des informations dans la liste de résultats du moteur de recherche.

\section{Le parfum : activation des catégories d'information fonctionnelle}

L'activation de l'information est examinée dans sa dimension fonctionnelle (Tableau 1), i.e. par rapport au but de l'usager. Elle traduit le découpage des unités ou séquences d'information significatives pour l'usager. La première stratégie repose sur une contextualisation de l'information à différents degrés qui s'effectue à travers un codage de l'information à partir des connaissances antérieures de l'usager et de l'information disponible dans l'environnement. Cette stratégie se caractérise par la production d'une multiplicité de buts intermédiaires reposant sur des évaluations multiples de l'information. On la retrouve par exemple dans le mode opératoire du chercheur, basé sur une combinaison de mots-clés reformulés tout au long du parcours. La deuxième stratégie a pour objectif l'identification d'un type de ressource correspondant aux préférences de l'usager. L'information utilisée s'apparente à une forme de signalisation que l'usager juge efficace pour construire une procédure en fonction de ses propres expériences. La stratégie se manifeste par l'utilisation d'informations successives qui permettent de répondre à un but intermédiaire unique chaque fois que l'usager traverse une grappe d'informations, i.e. un déroulement chronologique et exclusif du but. Une illustration est donnée par le mode opératoire des ingénieurs pédagogiques qui définissent a priori le genre et la durée de la vidéo en fonction du public-cible pour lequel ils font des recherches. Enfin, la troisième stratégie rencontrée est directement construite à partir des conditions d'usage prévues par l'usager qui génèrent un besoin de services. Elle se base sur un raisonnement prospectif (Richard, 1990) qui repose sur le besoin de trouver une information unique qui traduira l'existence ou non dans l'environnement du service souhaité. Cette information conditionne la satisfaction du but dès la prise en charge de la recherche et ne génère aucun but intermédiaire, à partir du moment où elle est connue. Un exemple est observé dans le mode opératoire d'un étudiant pour qui le téléchargement est la condition sine qua non de sélection d'une vidéo.

Tableau 1. Catégories d'informations fonctionnelles. Table 1. Categories of functional information

\begin{tabular}{|l|l|l|}
\hline Stratégies & Fonction de l'information & $\begin{array}{l}\text { Exemple d'information } \\
\text { mentionnée par les usagers }\end{array}$ \\
\hline $\begin{array}{l}\text { Basée sur des } \\
\text { connaissances }\end{array}$ & $\begin{array}{l}\text { Besoin de contextualiser l'information } \\
\text { disponible }\end{array}$ & $\begin{array}{l}\text { Combinaison de mots-clés et } \\
\text { d'informations diverses }\end{array}$ \\
\cline { 2 - 3 } & Référence de l'intervenant & Auteur \\
\cline { 2 - 3 } & Recherche d'un contenu actualisé & Date de réalisation \\
\cline { 2 - 3 } $\begin{array}{l}\text { Basée sur les } \\
\text { procédures }\end{array}$ & Vérification de la source & Producteur \\
\hline \multirow{2}{*}{$\begin{array}{l}\text { Basée sur le } \\
\text { raisonnement prospectif } \\
\text { (conditions d'usage) }\end{array}$} & Reche de latégorie de contenu & Genre de la vidéo \\
\cline { 2 - 3 } & Recherche du format & Durée de la vidéo \\
\cline { 2 - 3 } & Guidage par le système & Possibilité de téléchargement \\
\hline
\end{tabular}


37 La représentation de la solution choisie par l'usager - reposant la plupart du temps sur une combinaison de ces types de stratégies - permet de transcrire une partie de son raisonnement.

\section{Modes de visionnage et services corrélés avec la notion de temps}

Les usagers déclarent que le visionnage d'une vidéo est conditionné par le temps dont ils disposent a priori. Différentes contraintes de temps se manifestent à travers le mode de visionnage choisi, en lien avec la disponibilité des services de téléchargement, le stockage et la segmentation sémantique de la vidéo (Tableau 2). Deux pratiques majoritaires ont été constatées. La première consiste à visionner une vidéo complète une seule fois (Linear viewing style, De Boer, Kommers, \& de Brock, 2011) en visionnage immédiat ou différé. Les services de téléchargement et de stockage s'avèrent alors particulièrement importants pour l'usager. La deuxième reprend la pratique précédente en réitérant le visionnage de segments sélectionnés (Strategic viewing style, ibid.). Lorsque la vidéo n'est pas segmentée, les visiteurs signalent qu'ils doivent noter le timing des moments pertinents de la vidéo pour pouvoir les retrouver ultérieurement.

Tableau 2. Temps investi, types de visionnage et services. Table 2. Time spent, types of viewing and services

\begin{tabular}{|l|l|l|}
\hline Mode de visionnage & Services associés & Temps investi \\
\hline Visionnage unique complet & Eventuellement stockage & Temps réel incompressible \\
\hline $\begin{array}{l}\text { Visionnage complet doublé de } \\
\text { certains segments }\end{array}$ & Chapitrage & Temps partiellement redoublé \\
\hline $\begin{array}{l}\text { Visionnage de certains segments } \\
\text { uniquement }\end{array}$ & Chapitrage & Restriction du temps \\
\hline Nouveau visionnage complet & Stockage & Temps redoublé \\
\hline Ecoute de l'audio uniquement & $\begin{array}{l}\text { Eventuellement } \\
\text { téléchargement audio }\end{array}$ & Temps parallèle ou simultané \\
\hline
\end{tabular}

39 Suivent deux pratiques moins systématiques qui correspondent à une restriction du temps pour l'une et à un temps redoublé pour l'autre. Tout d'abord le visionnage sélectif de certains passages choisis (Maintenance rehearsal viewing style, ibid.). Cette pratique se manifeste si la vidéo se révèle moins intéressante que ce que le visiteur avait estimé ou s'il cherche à évaluer le contenu avant le stockage pour un visionnage ultérieur. La dernière pratique correspond au visionnage multiple d'une vidéo complète (Elaboration viewing style, ibid.). Elle survient lorsque le contenu d'une vidéo s'avère tout particulièrement pertinent. Les visiteurs signalent alors le besoin de stocker la vidéo ou de mémoriser le chemin pour la retrouver rapidement.

On a enfin relevé une situation marginale, conditionnée par la nature du contenu de la vidéo: la pratique du multitâche. Certains expliquent que la vidéo peut engendrer un temps incompressible qui ne permet pas de faire autre chose en même temps, notamment lorsqu'il est nécessaire de se concentrer sur l'image pour comprendre le discours (par exemple lorsque l'intervenant commente un Powerpoint). Cependant, d'autres au contraire expliquent que la vidéo peut apporter une flexibilité de consultation lorsqu'il est possible de dissocier le son de l'image. On a alors affaire à un temps parallèle ou simultané qui peut être mis à profit pour faire autre chose (par exemple «effectuer des recherches complémentaires sur un auteur ») ou qui favorise des conditions particulières de visionnage (par exemple « dans les transports »). Ils comparent volontiers cette pratique à 
l'écoute de la radio et ajoutent que pour les conférences filmées ou les interviews, " l'image n'a bien souvent aucun intérêt de toute façon ».

\subsection{Diversité des buts rencontrés}

41 Ce chapitre restitue la singularité des modes opératoires pour chaque catégorie de profils rencontrés, obtenue en regroupant les usagers selon leur but. Parmi les usagers interrogés, ceux qui fréquentent régulièrement le dispositif audiovisuel et recherchent fréquemment des vidéos sont soit des usagers qui cherchent à transmettre une ressource à autrui, soit des usagers «à distance » qui préparent un travail universitaire, soit des usagers qui viennent se cultiver.

\section{Trouver une ressource pour ses étudiants ${ }^{14}$}

42 Le premier but recensé concerne une recherche pour autrui, motivée par un objectif pédagogique. Elle correspond à un besoin immédiat, connu a priori et engendre un but très précis. Le profil ne cherche pas une information mais une ressource qu'il pourra transmettre, dont la pertinence repose sur une série de critères spécifiques qui devront tous être satisfaits pour que la ressource soit sélectionnée. Ces critères correspondent à un raisonnement prospectif répondant au niveau du public ciblé, à une thématique qui favorisera la motivation de la cible et à la satisfaction d'un objectif d'apprentissage (par exemple le niveau de langue recherché pour des étudiants en FLE). Le temps investi $a$ priori dans la recherche est très limité car l'usager préfère investir le temps dont il dispose dans des traitements pédagogiques (par exemple la transcription d'une partie du discours ou la production d'exercices). L'accès utilisé est le moteur de recherche avancée et ses possibilités de filtrer les critères. La ressource sera sélectionnée dès la liste de résultats du moteur de recherche, la notice ne sera donc généralement pas lue. Les premières minutes de la vidéo peuvent être écoutées dans certains cas pour s'assurer de l'adéquation entre la difficulté de la langue et le public visé. L'usager s'impose la contrainte de trouver une ressource pertinente dans un temps de recherche court. Si cette condition est satisfaite, le rapport coût / bénéfice est estimé positif pour la règle choisie par l'usager, sinon il ne réitère pas sa recherche et abandonne le dispositif pour un autre.

\section{Préparer un travail universitaire}

Pour le deuxième but rencontré, la recherche d'information est motivée par le besoin d'apprendre et par la recherche de concepts, de notions et de références réutilisables dans une production universitaire. Elle s'inscrit dans une recherche d'information plus vaste qui inclut des sources multiples et des documents hétérogènes. La vidéo apporte une flexibilité de consultation et permet d'obtenir de l'information «gratuitement, librement et à distance ». Elle correspond à un temps long, borné par une production, pendant lequel les recherches se réitèrent de nombreuses fois. L'étudiant 1 déclare avoir reçu une formation sur la recherche documentaire avancée, l'étudiant 2 avoue "ne pas avoir de stratégie de recherche consciente ». Le choix d'un accès sur la page d'accueil est motivé par l'impression générale de la page, par la compréhension du fonctionnement du système mais également par des habitudes de recherche qui reposent sur l'utilisation du moteur de recherche sur tous les sites qu'ils consultent. Au moment de la prise en charge, 
ils produisent un seul mot-clé correspondant à leur sujet ou à leur thématique de travail. On constate alors plusieurs types de stratégies. L'étudiant 1 suit une stratégie procédurale qui repose sur un pari puisque chaque recherche débutera par le même mot-clé et sera réitérée en espérant qu'une ressource apparaisse un jour. La stratégie de l'étudiant 2 correspond à un raisonnement prospectif qui s'appuie sur la possibilité de téléchargement, condition sine qua non du visionnage et sur une durée de la vidéo supérieure à 20 minutes, gage de "sérieux». Sur la notice, les deux étudiants déclarent apprécier une information visuelle et vérifient la conformité des informations déjà évaluées au niveau supérieur. L'étudiant 1 précise qu'il inspecte l'ensemble de la page en incluant les commentaires et les vidéos similaires. Les deux étudiants déclarent utiliser la segmentation sémantique de la vidéo et les documents d'accompagnement pour obtenir une représentation de la structure du contenu de la vidéo et écoutent généralement les premières minutes de la vidéo pour confirmer «l'orientation du contenu ». Pendant ou après le visionnage, ils font des recherches complémentaires, sur «les pages personnelles des auteurs " par exemple. La relation temps long disponible / domaine ciblé rend le dispositif efficace pour l'usager dans le contexte de son activité.

\section{Se cultiver ${ }^{15}$} ne repose pas sur une situation initiale motivée par un besoin d'information, ni sur un objectif final précis, mais sur un but flou et sans contrainte. Cette pratique s'inscrit dans un temps oisif, étiré, volontairement non maîtrisé, pendant lequel l'usager «navigue par plaisir » et consulte des vidéos dans des domaines variés. Il apprécie les dispositifs audiovisuels car ils "centralisent une diversité de points de vue » et affirme que la vidéo " nécessite moins d'effort que la lecture ». Ce profil «accepte ce qu'il reçoit parce que l'enjeu n'est pas de préparer un travail précis ». Il déclare perdre son temps si le fonds audiovisuel ne se renouvelle pas suffisamment et avoir rapidement "l'impression de tourner en rond" lorsqu'il consulte les collections. Après le visionnage, il poursuit généralement sa séance en sélectionnant une "vidéo similaire». L'ensemble des profils rencontrés déclare visionner des vidéos de temps en temps pour se cultiver (sauf l'enseignant et un ingénieur pédagogique : deux buts qui se déroulent dans un contexte pédagogique et qui correspondent à des recherches pour autrui).

\subsection{Conclusion de l'étude 2}

Le découpage de l'activité ainsi proposé permet de s'intéresser à chaque phase identifiée, soit de façon autonome, i.e. en restant dans le périmètre de la tâche (par exemple celle correspondant à l'usage des « substituts " pour Marchionini) soit dans leurs relations, i.e. d'un niveau à un autre.

Parmi les buts recensés, on constate que les variables (Tableau 3) correspondent majoritairement à la nature du but (professionnel ou non), à un besoin d'information pour soi ou pour autrui (Tricot, 2015), à la nature et à la précision de la ressource ou de l'information recherchée, au temps investi (Pirolli, \& Card, 1999) et au type d'usage prévu (solution concrète, apprentissage, publication, etc.) qui conditionne le mode de visionnage a priori. 
Tableau 3. Description des buts et de leurs critères significatifs. Table 3. Description of the goals and their significant criteria

\begin{tabular}{|c|c|c|c|}
\hline Buts & Critères significatifs du but & $\begin{array}{l}\text { Investissement du } \\
\text { temps }\end{array}$ & Modes de visionnage \\
\hline $\begin{array}{l}\text { Trouver des } \\
\text { ressources } \\
\text { pédagogiques } \\
\text { pour autrui }\end{array}$ & $\begin{array}{l}\text { Recherche pour autrui. But } \\
\text { immédiat et précis, connu avant } \\
\text { la recherche. Ressource traitée } \\
\text { dans un contexte pédagogique }\end{array}$ & Limité & Généralement aucun \\
\hline $\begin{array}{l}\text { Préparer un } \\
\text { travail } \\
\text { universitaire }\end{array}$ & $\begin{array}{l}\text { But en cours de précision. } \\
\text { Construction de connaissances } \\
\text { sur un sujet en vue d'une } \\
\text { production }\end{array}$ & $\begin{array}{l}\text { Réitératif sur une } \\
\text { période } \\
\text { universitaire } \\
\text { bornée par une } \\
\text { production }\end{array}$ & $\begin{array}{l}\text { Temps réel ou différé, } \\
\text { multiple (Stockage) }\end{array}$ \\
\hline Se cultiver & $\begin{array}{l}\text { Besoin connu plutôt a posteriori } \\
\text { ou pendant la recherche. } \\
\text { Acquérir des connaissances sur } \\
\text { des domaines variés }\end{array}$ & Indéterminé & $\begin{array}{l}\text { Visionnage immédiat et } \\
\text { complet }\end{array}$ \\
\hline $\begin{array}{l}\text { Satisfaire des } \\
\text { services } \\
\text { pédagogiques: } \\
\text { veille, conseils, } \\
\text { trouver des } \\
\text { solutions } \\
\text { concrètes, etc. }\end{array}$ & $\begin{array}{l}\text { Recherche de ressources, } \\
\text { d'informations ou de solutions, } \\
\text { pour soi ou pour autrui. }\end{array}$ & $\begin{array}{l}\text { Plutôt court mais } \\
\text { peut être long } \\
\text { selon les objectifs }\end{array}$ & $\begin{array}{l}\text { Fractionné et sélectif, ou } \\
\text { complet selon le but. } \\
\text { Immédiat parfois différé }\end{array}$ \\
\hline $\begin{array}{l}\text { Préparer un } \\
\text { travail } \\
\text { scientifique }\end{array}$ & $\begin{array}{l}\text { Besoin immédiat, but précis } \\
\text { connu avant la recherche. } \\
\text { Information précise et } \\
\text { expertisée }\end{array}$ & $\begin{array}{l}\text { Plus ou moins long } \\
\text { selon le temps } \\
\text { initial disponible }\end{array}$ & Non usage \\
\hline
\end{tabular}

47 Les buts décrits dans cette étude correspondent à des usages fréquents de la vidéo. Cependant nous avons également rencontré deux profils (consignés dans le tableau cidessus) qui témoignent d'un usage sous conditions. Le premier correspond à une activité de services d'ingénierie pédagogique qui a besoin de ressources abondantes, diversifiées et régulièrement actualisées. Le manque de fonctionnalités précises de recherche et des vidéos mal adaptées aux besoins de l'Enseignement Supérieur selon eux, modèrent l'intégration de la vidéo dans leur activité professionnelle. Le deuxième but a pour objectif la préparation de communications scientifiques. Le chercheur estime que les collections audiovisuelles morcèlent le savoir et qu'il manque des outils pour structurer les relations entre les contenus. Il fait une analogie négative avec d'autres dispositifs familiers proposant notamment un moteur de recherche plus performant et des services d'indexation complexe adaptés à sa recherche d'expert. Il reconnaît cependant fréquenter parfois les dispositifs audiovisuels pour se cultiver.

\subsection{Conclusion générale}

Ces deux études permettent de mettre en évidence l'impact des contraintes, du périmètre et de la qualité de réalisation de la tâche de publication audiovisuelle sur l'activité de recherche de vidéo. 
Tableau 4. Périmètre et impacts de réalisation de la tâche de publication sur la consultation. Table 4. Scope and impacts of the publication task on consultation

\begin{tabular}{|l|l|l|l|l|}
\hline $\begin{array}{l}\text { Tâches de } \\
\text { publication }\end{array}$ & $\begin{array}{l}\text { Déclarations des non } \\
\text { documentalistes }\end{array}$ & $\begin{array}{l}\text { Déclarations des } \\
\text { documentalistes }\end{array}$ & Tâches de consultation & Déclarations des visiteurs \\
\hline $\begin{array}{l}\text { Encodage } \\
\text { (technique) }\end{array}$ & $\begin{array}{l}\text { Incompréhension } \\
\text { parfois de la finalité de } \\
\text { la tâche }\end{array}$ & $\begin{array}{l}\text { Incompréhension parfois } \\
\text { de la finalité de la tâche }\end{array}$ & Visionnage & $\begin{array}{l}\text { Difficultés liées aux qualités de } \\
\text { connexion }\end{array}$ \\
\hline $\begin{array}{l}\text { Indexation } \\
\text { (éditorial) }\end{array}$ & $\begin{array}{l}\text { Difficulté verbalisée } \\
\text { qui n'empêche pas la } \\
\text { réalisation de la tâche }\end{array}$ & Compétence-métier & $\begin{array}{l}\text { Identification de la } \\
\text { vidéo (données et } \\
\text { métadonnées) }\end{array}$ & $\begin{array}{l}\text { Difficultés dans la confirmation de la } \\
\text { sélection de la ressources, l'évaluation } \\
\text { du contenu et/ou sa } \\
\text { crédibilité/fiabilité }\end{array}$ \\
\hline $\begin{array}{l}\text { Classement } \\
\text { (documentaire) }\end{array}$ & $\begin{array}{l}\text { Difficulté à exécuter la } \\
\text { tâche }\end{array}$ & Compétence-métier & $\begin{array}{l}\text { Appréhension de } \\
\text { l'organisation du } \\
\text { fonds, et des } \\
\text { ressources }\end{array}$ & $\begin{array}{l}\text { Incohérence de la logique de } \\
\text { classement entraînant une mauvaise } \\
\text { localisation des ressources }\end{array}$ \\
\hline $\begin{array}{l}\text { Organisations des } \\
\text { connaissances } \\
\text { (expertise du } \\
\text { domaine) }\end{array}$ & $\begin{array}{l}\text { Impossibilité de } \\
\text { réaliser la tâche }\end{array}$ & $\begin{array}{l}\text { Difficulté à réaliser la } \\
\text { tâche }\end{array}$ & $\begin{array}{l}\text { Compréhension de la } \\
\text { structuration des } \\
\text { connaissances du } \\
\text { domaine et de ses } \\
\text { thématiques }\end{array}$ & $\begin{array}{l}\text { Incompréhension de la logique de } \\
\text { regroupement des ressources et du } \\
\text { nommage des collections et de leurs } \\
\text { heuristiques }\end{array}$ \\
\hline
\end{tabular}

Les difficultés rencontrées peuvent entraîner un investissement du temps et un effort important dans la navigation et la compréhension de l'environnement, au lieu de permettre à l'usager de centrer son attention sur l'évaluation de la pertinence de l'information ou de la ressource par rapport à son but.

\section{Discussion}

L'objectif de cette recherche était de décrire deux activités centrées sur la vidéo académique en ligne : la publication audiovisuelle et la recherche de vidéo. L'étude a été effectuée en produisant tout d'abord une référence préalable à travers des graphes de la tâche a priori issus d'une inspection heuristique de l'outil de publication et de la plateforme en ligne, puis une étude des usages réels en interrogeant des opérateurs responsables de la publication audiovisuelle sur le dispositif étudié ainsi que des usagers familiers de ce même dispositif.

51 Les résultats mettent en évidence le fait que la décision de fréquenter un dispositif audiovisuel répond à la préférence de l'usager pour le média vidéo, à la volonté de fournir un effort moindre que la lecture ou encore à la recherche d'une connaissance uniquement disponible dans ce type de dispositif. La définition des critères de pertinence de la vidéo recherchée est directement corrélée avec le besoin d'information : soit l'usager recherche une ressource audiovisuelle qui sera traitée et transmise à un tiers, soit il recherche de l'information destinée à être citée, à illustrer un propos ou encore à favoriser la construction de connaissances sur un thème précis. Une situation marginale a cependant été constatée lorsque l'usager fréquente le dispositif pour se cultiver. L'usager se déclare alors incapable de décrire son besoin d'information, son but ou la thématique dans laquelle s'inscrit sa recherche.

52 Les résultats ont produit un découpage de l'activité de consultation de vidéo qui peut être mis en perspective avec l'activité de publication audiovisuelle à travers l'organisation du fonds, la structuration des connaissances, l'indexation, l'évaluation du contenu et de sa structure et les services destinés à faciliter les usages prévus de la vidéo (Cf. Tableau 4). 

pour mettre en ligne une vidéo. La première tâche concerne l'encodage du fichier vidéo. Les résultats montrent que les documentalistes et les non-documentalistes seraient encouragés dans la réalisation de la tâche par une explicitation de sa finalité, i.e. son impact sur les conditions de visionnage de l'usager final. La deuxième tâche est double. Elle concerne tout d'abord une indexation obligatoire destinée à produire une notice minimale garantissant les conditions d'identification de la ressource et l'évaluation de sa crédibilité par l'usager. La question du périmètre et de la nature des données qui doivent être produites conditionne également la possibilité de redistribuer ces informations dans l'environnement afin de favoriser le suivi du parfum (Pirolli, \& Card, 1999), i.e. tant l'efficacité de l'information (sa localisation) que sa précision (nature de l'information). Pour les non-documentalistes, la tâche minimale d'indexation représente la finalité de leur activité. Elle a pour objectif une réalisation rapide de la tâche qui repose sur le report des informations qui leur sont transmises initialement avec le fichier vidéo. d'information et que l'exactitude et la complétude des données relèvent de la responsabilité des auteurs, producteurs, organisateurs, etc.

Pour les documentalistes, à l'inverse, la tâche d'indexation minimale représente le début de leur activité. Leur attention se porte sur la performance de la tâche qui se traduit par la production d'une notice basée sur des recherches complémentaires qui leur permettent de vérifier et de compléter les informations qu'ils transmettent à l'usager. La deuxième partie de la tâche concerne une indexation complexe qui ne peut être associée qu'à des statuts flexibles (ceux pour lesquels l'opérateur possède une réelle liberté - Chevalier, \& Tricot, 2008) car elle implique à la fois des compétences-métier et un degré de réalisation conditionné par le temps disponible. Les documentalistes sont les seuls à effectuer cette tâche qui se traduit d'une part, par la production de substituts textuels - un résumé basé sur des notes prises pendant le visionnage de la vidéo et des mots-clés qui complètent, selon eux, les autres types de classement - et d'autre part, par une segmentation sémantique de la vidéo qui permettra par exemple au lecteur d'évaluer la structure du contenu au moment de la sélection. Les documentalistes signalent le besoin de services éditoriaux plus développés dans l'outil de publication qui leur permettraient de répondre aux spécificités de leurs corpus, à celles du média vidéo et aux attentes d'usagers ciblés. La troisième tâche concerne les différents types de classement. Les non-documentalistes mentionnent une difficulté à réaliser la tâche et une incapacité à la réguler qui viendrait d'une impossibilité à autoévaluer leur travail. Alors que les non-documentalistes décrivent une réalisation de tâches successives et indépendantes les unes des autres, les documentalistes, envisagent l'activité dans sa globalité en décrivant des tâches complémentaires. Ils signalent le risque d'incohérence qui peut résulter d'un manque de pertinence dans le choix du type de classements pour l'usager final, l'inadéquation à certains types de corpus et le risque d'une indexation collective non maitrisée. Enfin, on a constaté une difficulté partagée concernant la conception des collections. Nous n'avons rencontré qu'un seul service dans lequel collaboraient un documentaliste et des nondocumentalistes. Dans les autres organisations, la publication audiovisuelle reposait, pour l'un d'entre eux, sur une équipe de non-documentalistes et pour les autres, sur une seule personne, documentaliste ou non-documentaliste. Les non-documentalistes déclarent que la création de leurs collections ne repose sur aucune conception mais plutôt sur un regroupement qui peut dupliquer celle de l'évènement ou de l'institution productrice ou 
encore sur une organisation « au fil de l'eau ». Ils déclarent ne pas avoir d'interlocuteur compétent pour les aider dans cette tâche. Les documentalistes émettent un questionnement sur l'intelligibilité de l'organisation des collections pour le visiteur, sans pour autant apporter de réponse satisfaisante selon eux.

L'étude de la consultation s'est effectuée à travers la relation entre les caractéristiques de l'environnement en ligne (comprenant le résultat de la tâche de publication), celles de l'usager et la réalisation effective de la tâche. La compréhension de la structuration du fonds par l'usager a été observée à travers la logique de classement des ressources, traduite par l'utilisation des accès de premier niveau sur la page d'accueil. On constate que des contraintes cognitives entraînent des actions plus réduites que la règle, d'une part, lorsque l'inférence par rapport aux accès proposés s'avère difficile (par exemple des entrées thématiques trop restreintes), d'autre part, lorsque l'usager doit produire un effort trop important de mémorisation (par exemple une mise en avant du producteur) ou encore lorsque ces accès de premier niveau sont trop abondants. Les usagers ne cherchent alors plus l'accès qui leur semble le plus pertinent mais celui qui s'avère le moins contraignant pour eux ou celui qui répond à leurs habitudes, i.e. celui qui leur permet de retrouver rapidement leurs repères. Dans cette étude, l'usage par défaut du moteur de recherche correspond très majoritairement à un renoncement à la consultation des collections qui représente pourtant la mise en valeur du travail des entités contributrices et la particularité de ce dispositif collaboratif. À l'intérieur des collections, les usagers témoignent d'une incompréhension de la structuration des connaissances, de la logique de regroupement des ressources ou encore du manque de clarté concernant le nommage des heuristiques.

On constate que les notices peuvent être consultées avant le visionnage de la vidéo pour confirmer ou infirmer une présélection, pendant le visionnage pour vérifier l'exactitude des informations proposées ou a posteriori pour relancer une recherche ou pour obtenir des informations supplémentaires. Dans cette étude, la tâche d'évaluation du contenu est effectuée par l'ensemble des usagers (excepté l'enseignant). Lorsque la vidéo n'est pas accompagnée de substituts (Marchionini, Song, \& Farrell, 2009), la contrainte est régulée par des tâches alternatives. Les substituts textuels traditionnels, comme le résumé ou les mots-clés, ne semblent effectivement pas suffisants pour la majorité des usagers. On constate une variété de tâches alternatives selon le but de l'usager, comme par exemple l'évaluation du contenu basé sur des informations audio, textuelles et parfois visuelles (Yang, \& Marchionini, 2004) ou encore l'évaluation de la structure du contenu à travers la lecture des documents d'accompagnement (notamment le Powerpoint) ou de la segmentation de la vidéo lorsqu'elle est sémantique. Ces actions étant très peu encouragées et pourtant réalisées, l'utilité et la plus-value d'un service éditorial complexe semblent effectivement pertinentes. La spécificité du média vidéo, chronophage a priori, entraîne un besoin de services liés à la manipulation de l'objet vidéo et à l'anticipation des conditions d'usage. D'après les usagers, la segmentation de la vidéo, même lorsqu'elle repose sur un découpage rapide des grandes parties de la vidéo, apporte une aide à la navigation dans le contenu et peut ainsi réduire les contraintes de temps liées au visionnage. D'autres services, comme le téléchargement (lié à une autorisation préalable des auteurs) ou les facilités de stockage, apportent une flexibilité de consultation en s'adaptant à la situation de certains usagers (par exemple un visionnage multiple sans procéder à une nouvelle recherche). 
58 Dans cette étude, deux difficultés semblent justifier l'abandon de la consultation des collections. La première relève de la responsabilité du dispositif à inciter l'usager à consulter les collections en mettant en valeur leur accès sur la page d'accueil et en s'assurant que l'usager puisse inférer rapidement du nommage des collections par rapport à son but - par exemple à travers une thématique intelligible - sans avoir à détenir des informations supplémentaires pour débuter sa recherche, comme le nom de l'institution productrice ou la date de production d'une ressource. La deuxième questionne le besoin d'expertise des chaînes audiovisuelles pour concevoir l'organisation des connaissances du domaine de leurs collections et leur intelligibilité pour l'usager.

Le manque de fiabilité collective de l'indexation réalisée mène l'usager final à devoir vérifier les informations produites, à les compléter et à s'assurer de leur adéquation au contenu de la vidéo. Les conditions et le degré de réalisation de la tâche de publication ont donc un impact important sur l'investissement du temps dans la recherche d'information de l'usager et sur l'estimation de son coût par rapport au gain obtenu (Pirolli, \& Card 1999) qui peut affecter la fréquentation du dispositif. La nature collaborative de la publication nécessite un contrôle accru de la fiabilité des données produites qui pourrait être accompagné par plus de guidage, de protection contre les erreurs, de feedback et de communication sur les enjeux de la tâche. Enfin, on constate une exigence de la part de certains usagers concernant les services éditoriaux attendus dans ces environnements académiques audiovisuels. Les attentes reposent sur une analogie avec des services qui leur sont familiers (par exemple des moteurs de recherche spécialisés) ou spécifiques à leur environnement professionnel habituel (l'enseignement ou la recherche notamment). L'ensemble des usagers s'attend également à trouver une expertise, i.e. d'une plus-value (par exemple dans l'organisation des connaissances du domaine). Pour satisfaire ces usagers, l'étendue de ces services semble devoir dépasser ceux que proposent d'autres types de dispositifs audiovisuels, non académiques, dont le corpus n'est pas ciblé et qui n'ont pas le même type d'ambition.

60 La diversité des modes opératoires rencontrés a abouti à la coexistence de deux catégories de buts de nature différentes pour l'activité de consultation de vidéos académiques en ligne. La première catégorie regroupe les buts issus d'une situation en résolution de problème pour lesquels les usagers initient une recherche pour trouver une ressource ou une information qui leur permettra de résoudre la situation (le but est lié à une activité professionnelle ou à un apprentissage dans un domaine ciblé). La deuxième catégorie repose sur des buts pour lesquels l'activité n'est plus secondaire mais se justifie par elle-même (le but poursuivi ici repose sur la volonté de se cultiver). Dans cette deuxième catégorie, le but peut être unique pour certains usagers et secondaire pour d'autres (i.e. qu'ils consultent parfois le dispositif avec un objectif professionnel et parfois pour se cultiver). Ce but semble prendre une place importante dans les environnements numériques destinés à la diffusion du savoir, or il reste sous-étudié. L'une des limites de cette étude réside dans la taille de l'échantillon interrogé. Une étude quantitative permettrait d'étendre et de préciser la description des principes fonctionnels des buts et des processus qui les caractérisent.

61 La plupart des modèles théoriques et analytiques de recherche d'information repose sur un besoin d'information qui entraîne une activité principale centrée sur l'action. Or lorsque les usagers fréquentent un dispositif pour se cultiver, la description du but ne correspond ni à une situation en résolution de problème, ni à une activité basée sur la réception de l'information centrée sur la compréhension (comme par exemple la lecture 
d'un roman). Le constat de la coexistence de ces buts de natures différentes dans un même environnement amène à s'interroger sur le modèle d'analyse à adopter pour leur étude conjointe.

\section{BIBLIOGRAPHIE}

Bachimont, B. (1999). Bibliothèques numériques audiovisuelles : des enjeux scientifiques et techniques. Document numérique, 2, 219-242.

Bastien, J. C., Leulier, C., \& Scapin, D. L. (1998). L'ergonomie des sites web. In J.C. Moal \& B. Hidoine (Eds), Créer et maintenir un service Web, (pp. 111-173). Paris : ADBS.

Bisseret, J., Sébillotte, S., \& Falzon, P. (1999). Techniques pratiques d'analyse des activités expertes. Toulouse : Octarès.

Chen, C. M., \& Wu, C. H. (2015). Effects of different video lecture types on sustained attention, emotion, cognitive load, and learning performance. Computers \& Education, 80, 108-121.

Chevalier, A., \& Cegarra, J. (2008). Une approche psychologique de la notion de contrainte en résolution de problèmes. Le Travail Humain, 71, 173-198.

Chevalier, A., \& Tricot, A. (2008). Ergonomie des documents électroniques. Paris : Presses Universitaires de France.

Cojean, S., \& Jamet, E. (2017). Facilitating information-seeking activity in instructional videos: The combined effects of micro-and macroscaffolding. Computers in Human Behavior, 74, 294-302.

Crozier, M., \& Friedberg, E. (1977). L'acteur et le système. Paris : Seuil.

De Boer, J., Kommers, P. A., \& De Brock, B. (2011). Using learning styles and viewing styles in streaming video. Computers \& Education, 56, 727-735.

Ding, W., Marchionini, G., \& Soergel, D. (1999). Multimodal surrogates for video browsing. In Proceedings of the fourth ACM conference on Digital libraries (pp. 85-93). New York: ACM.

Falzon, P. (1994). Les activités méta-fonctionnelles et leur assistance. Le Travail Humain, 57, 1-23.

Ganier, F., \& De Vries, P. (2016). Are instructions in video format always better than photographs when learning manual techniques? The case of learning how to do sutures. Learning and Instruction, 44, 87-96.

Greene, S., Marchionini, G., Plaisant, C., \& Shneiderman, B. (2000). Previews and overviews in digital libraries: Designing surrogates to support visual information seeking. Journal of the Association for Information Science and Technology, 51, 380-393.

Heilesen, S. B. (2010). What is the academic efficacy of podcasting? Computers \& Education, 55, 1063-1068.

Hoc, J. M., \& Leplat, J. (1983). Evaluation of different modalities of verbalization in a sorting task. International Journal of Man-Machine Studies, 18, 283-306. 
Hughes, A., Wilkens, T., Wildemuth, B. M., \& Marchionini, G. (2003). Text or pictures? An eyetracking study of how people view digital video surrogates. In E. M. Bakker et al. (Eds). CIVR 2003, LNCS 2728, (pp. 271-280). Berlin Heidelberg : Springer-Verlag.

Kay, R. H. (2012). Exploring the use of video podcasts in education: A comprehensive review of the literature. Computers in Human Behavior, 28, 820-831.

Kousha, K., Thelwall, M., \& Abdoli, M. (2012). The role of online videos in research communication: A content analysis of YouTube videos cited in academic publications. Journal of the Association for Information Science and Technology, 63, 1710-1727.

Leplat, J. (2015). Repères pour l'analyse de l'activité en ergonomie. Paris : Presses Universitaires de France.

Leplat, J. (2006). La notion de régulation dans l'analyse de l'activité. Pistes, 8, 1-25. http:// pistes.revues.org/3101; DOI : 10.4000/pistes.3101

Marchionini, G., Song, Y., \& Farrell, R. (2009). Multimedia surrogates for video gisting: Toward combining spoken words and imagery. Information Processing \& Management, 45, 615-630.

Marchionini, G., Wildemuth, B. M., \& Geisler, G. (2006). The open video digital library: a Möbius strip of research and practice. Journal of the Association for Information Science and Technology, 57, 1629-1643.

Marchionini, G., Geisler, G., \& Brunk, B. (2000). Agile views: A human-centered framework for interfaces to information spaces. In Proceedings of the ASIST Annual Meeting (Vol. 37, pp. 271-280).

Merkt, M., \& Schwan, S. (2014). Training the use of interactive videos: effects on mastering different tasks. Instructional Science, 42, 421-441.

Pirolli, P. (2007). Information foraging theory: Adaptive interaction with information. Oxford: Oxford University Press.

Pirolli, P., \& Card, S. (2005). The sensemaking process and leverage points for analyst technology as identified through cognitive task analysis. In Proceedings of international conference on intelligence analysis (Vol. 5, pp. 2-4).

Pirolli, P., \& Card, S. (1999). Information foraging. Psychological Review, 106, 643-675.

Rasmussen, J. (1983). Skills, rules, and knowledge; signals, signs, and symbols, and other distinctions in human performance models. IEEE transactions on systems, man, and cybernetics, (3), 257-266.

Richard, J. F. (1990). Les activités mentales. Comprendre, raisonner, trouver des solutions. Paris : Armand Colin.

Robinson, O. C. (2014). Sampling in interview-based qualitative research: A theoretical and practical guide. Qualitative Research in Psychology, 11(1), 25-41.

Sebillotte, S. (1994). Méthodologie pratique d'analyse de la tâche en vue de l'extraction de caractéristiques pertinentes pour la conception d'interfaces. Rapports techniques- I. N. R. I. A. Sebillotte, S. (1991). Décrire des tâches selon les objectifs des opérateurs, de l'interview à la formalisation. Le Travail Humain, 54, 193-223.

Schoeffmann, K., Hopfgartner, F., Marques, O., Boeszoermenyi, L., \& Jose, J. M. (2010). Video browsing interfaces and applications: a review. Journal of Photonics for Energy, 018004-018004. 
Shearer, E., \& Gottfried, J. (2017). News Use Across social Media Platforms 2017, Pew research Center, September. Retrieved: http: //assets.pewresearch.org/wp-content/uploads/ sites/13/2017/09/13163032/PJ_17.08.23_socialMediaUpdate_FINAL.pdf

Song, Y., \& Marchionini, G. (2007). Effects of audio and visual surrogates for making sense of digital video. In Proceedings of the SIGCHI Conference on Human Factors in Computing Systems (pp. 867-876). New York: ACM.

Sullivan, J. R. (2012). Skype: An appropriate method of data collection for qualitative interviews? The Hilltop Review, 6(1), 10.

Tricot, A. (2015). Besoin d'information. In Dictionnaire des concepts en soins infirmiers. Paris : Editions Setes.

Tricot, A. (2005). De l'intelligence à l'expertise, les effets de la diversité. P. Léna \& B. AjchenbaumBoffety, sous l'égide de l'Académie des Sciences (Eds.), Éducation, sciences cognitives et neurosciences (pp. 161-178). Paris : PUF.

Tricot, A., \& Nanard, J. (1998). Un point sur la modélisation des tâches de recherche d'informations dans le domaine des hypermédias. Les hypermédias, approches cognitives et ergonomiques, hors- série, 35-56.

Wildemuth, B., Marchionini, G., Wilkens, T., Yang, M., Geisler, G., Fowler, B., \& Mu, X. (2002). Alternative surrogates for video objects in a digital library: users' perspectives on their relative usability. Research and Advanced Technology for Digital Libraries, 143-160.

Yang, M., \& Marchionini, G. (2004). Exploring users' video relevance criteria - A pilot study. Proceedings of the Association for Information Science and Technology, 41, 229-238.

Yang, M., Wildemuth, B. M., Marchionini, G., Wilkens, T., Geisler, G., Hughes, A., \& Webster, C. (2003). Measuring user performance during interactions with digital video collections. Proceedings of the Association for Information Science and Technology, 40, 3-12.

\section{ANNEXES}

\section{Annexe 1 - Graphe de la tâche a priori de publication}

L'analyse de la tâche a priori repose sur une volonté de rendre visible « ce qui doit être fait " pour réaliser la tâche de publication audiovisuelle en ligne via l'outil de publication $\mathrm{du}$ dispositif. Une première intention était de définir le concept de « publication audiovisuelle en ligne » au centre de laquelle se trouve « l'objet-vidéo ». 
Graphe 2 : Tâche a priori N 1 « Publier une vidéo ». Graph 2 : A priori task $N^{\circ} 1$ « Post a video»

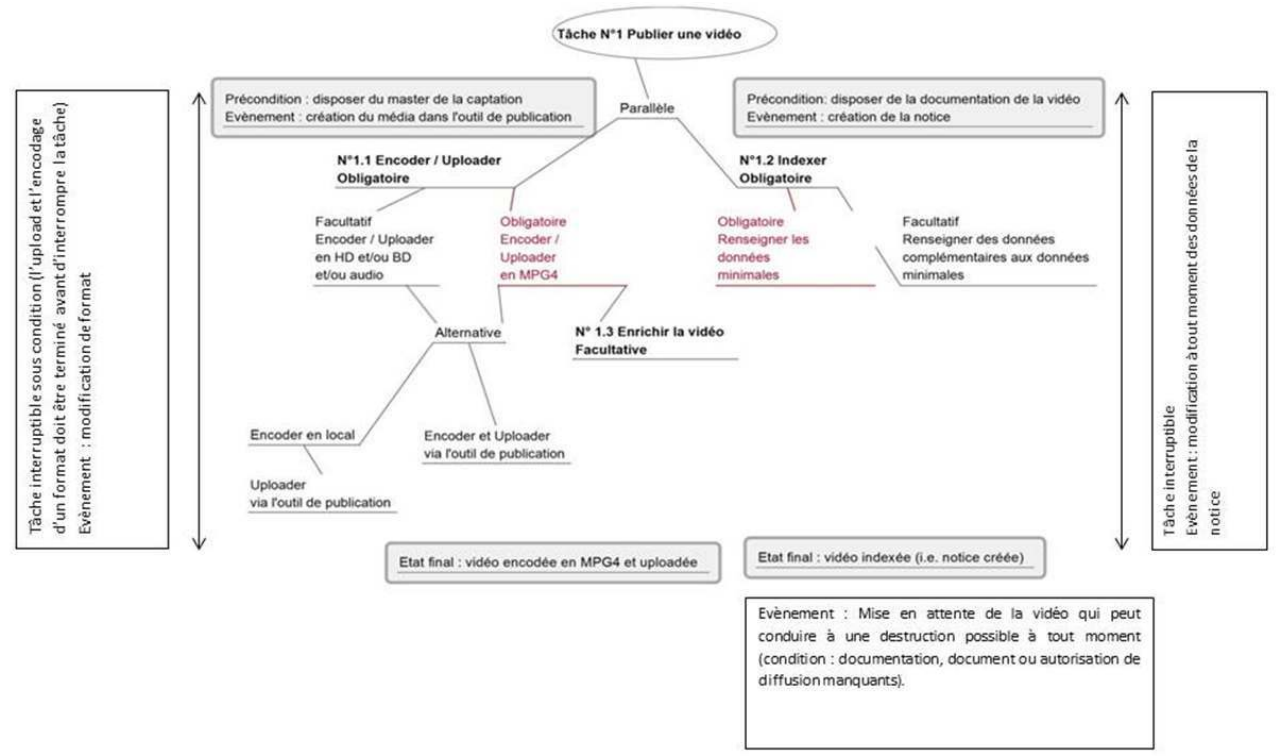

\section{Annexe 2 - Graphe de la tâche effective de publication}

L'analyse de la tâche effective est l'étude de « ce qui réellement effectué » par l'opérateur motivé par ses objectifs propres et sa logique.

Graphes des modes opératoires types incluant la répartition des tâches de publication

Les graphes ont permis d'élaborer une typologie des modes opératoires, comprenant la répartition des tâches entre opérateurs. Cette analyse a fait apparaître deux cas-types de contribution :

Cas-type où la tâche de publication audiovisuelle est à la charge d'une seule personne

L'opérateur unique est alors à la fois le responsable de la publication et de la chaîne. Les différences observables entre les graphes ci-dessous résident dans la déclinaison des sous-tâches de la tâche « $\mathrm{N}^{\circ} 1$ Publier une vidéo ». 
Graphe 3 - Type de mode opératoire 1 (non documentaliste).

Graph 3 : Procedure type 1 (non-documentalist)

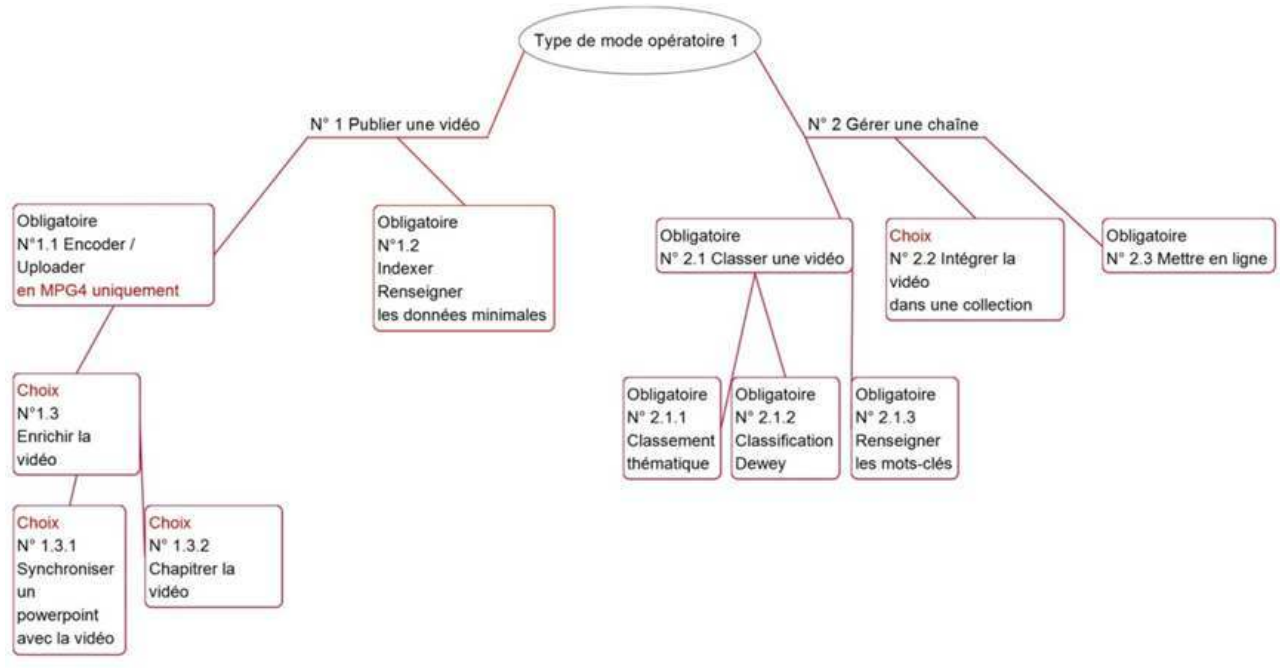

Graphe 4 : Type de mode opératoire 2 (documentaliste).

Graph 4 : Procedure type 2 (documentalist)

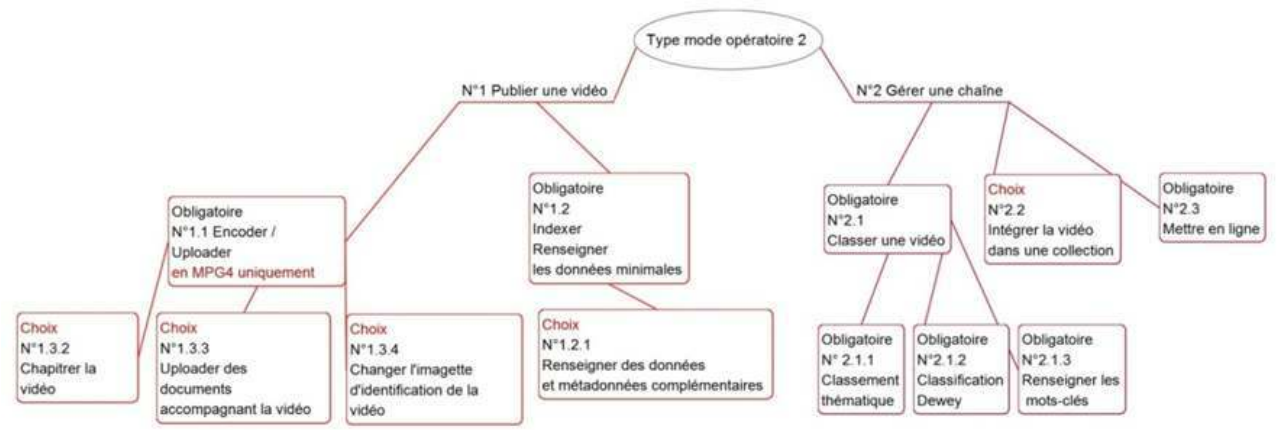

Cas-type où 2 opérateurs au moins participent à la réalisation de la tâche de publication audiovisuelle. Certains rôles sont parfois doublés dans le même service.

Graphe 5 : Mode opératoire 3 (plusieurs opérateurs non documentalistes).

Graph 5 : Procedure 3 (several non-documentalist operators)

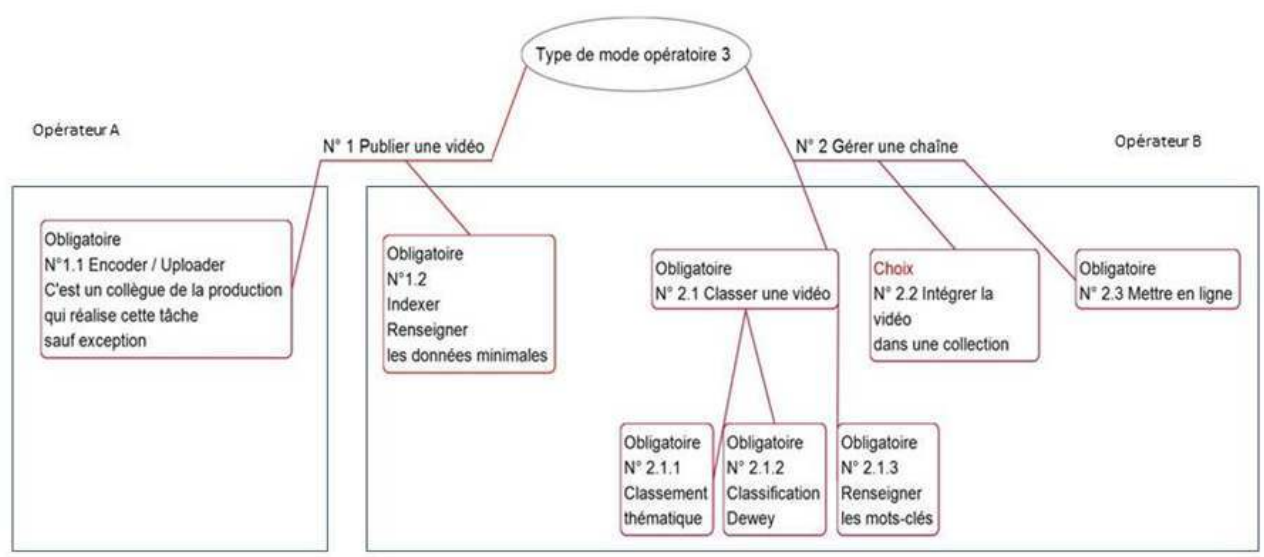


Dans ce cas de figure, l'opérateur B est responsable de la publication, il n'a pas un profil de documentaliste et il n'y a aucune supervision ni validation supplémentaire de la chaîne. Sa pratique s'apparente à celle des deux modes précités, à la seule différence que la tâche d'encodage et d'uploade est à la charge d'une tierce personne. Parfois ce n'est pas lui qui effectue la publication (l’opérateur ne souhaite pas préciser ce sujet).

Graphe 6 : Mode opératoire 4 (plusieurs opérateurs dont un documentaliste). Graph 6 : Procedure 4 (several operators including a documentalist)

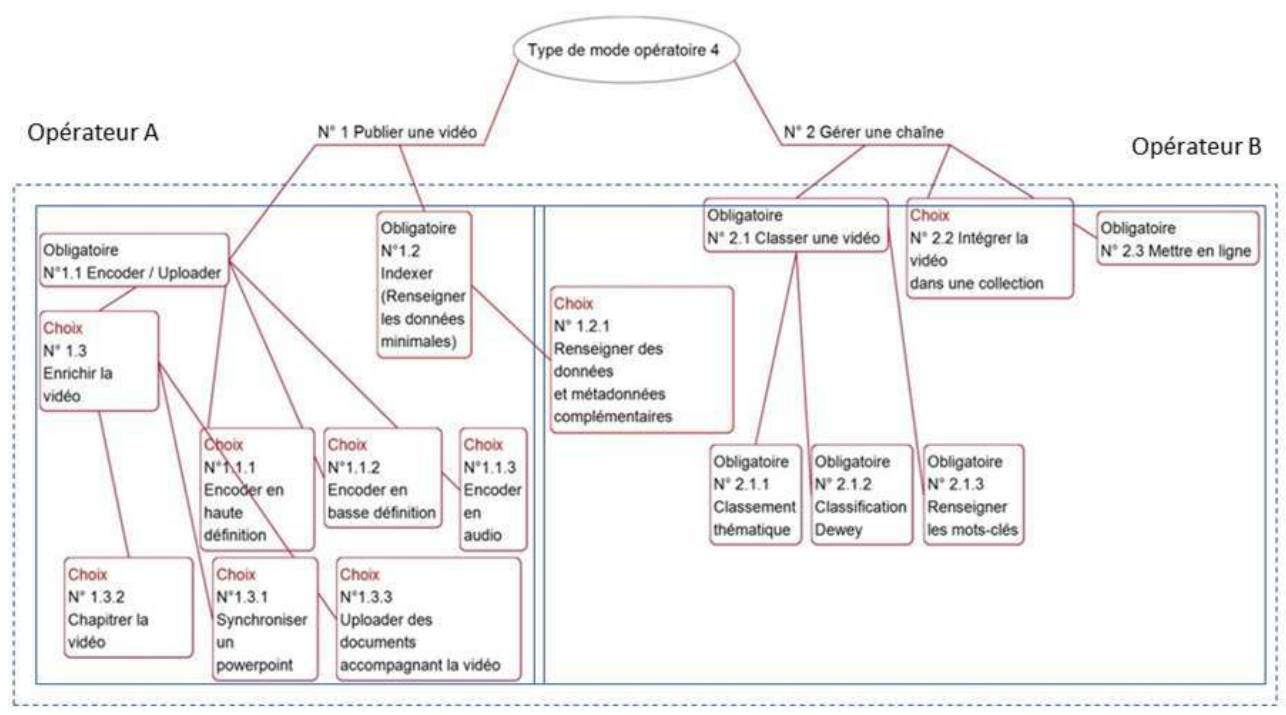

L'opérateur A (technicien audiovisuel) effectue la tâche $\mathrm{N}^{\circ} 1$ puis l'opérateur $\mathrm{B}$ (documentaliste) supervise et valide le travail de A avant d'effectuer la tâche $\mathrm{N}^{\circ} 2$. Il arrive parfois dans certains cas que l'opérateur B effectue l'ensemble des deux tâches. Parfois l'opérateur A réalise également l'ensemble de la publication, mais toujours sous la supervision de l'opérateur B. Ce modèle présente une répartition des rôles basée sur un périmètre affirmé de compétences distinguées. Cette équipe est composée de deux techniciens de l'image et du son qui se chargent des captations réalisées à l'intérieur de l'institution et de leur mise en ligne. Le troisième opérateur prend également en charge la sélection des captations et la réception de captations extérieures à leur service qu'il met alors en ligne lui-même. Il est d'une façon plus générale responsable de la vidéothèque dans ce cadre, il supervise, complète et valide le travail de ses deux collègues - et participe à l'ensemble des évènements audiovisuels de l'institution.

Graphe de la tâche effective commune de publication

Le graphe ci-dessous présente les tâches effectuées systématiquement par l'ensemble des opérateurs interrogés. Les éléments qui y sont consignés deviennent des tâches obligatoires. On remarquera que le graphe obtenu est en tous points similaires aux graphes de la tâche a priori, à l'exception de la tâche « Intégrer une vidéo dans une collection ». Facultative pour le système, on a pu constater que cette tâche était systématiquement réalisée par l'ensemble des opérateurs. 
Graphe 7 : Tâche effective commune à l'ensemble des opérateurs. Graph 7 : Effective task common to all operators

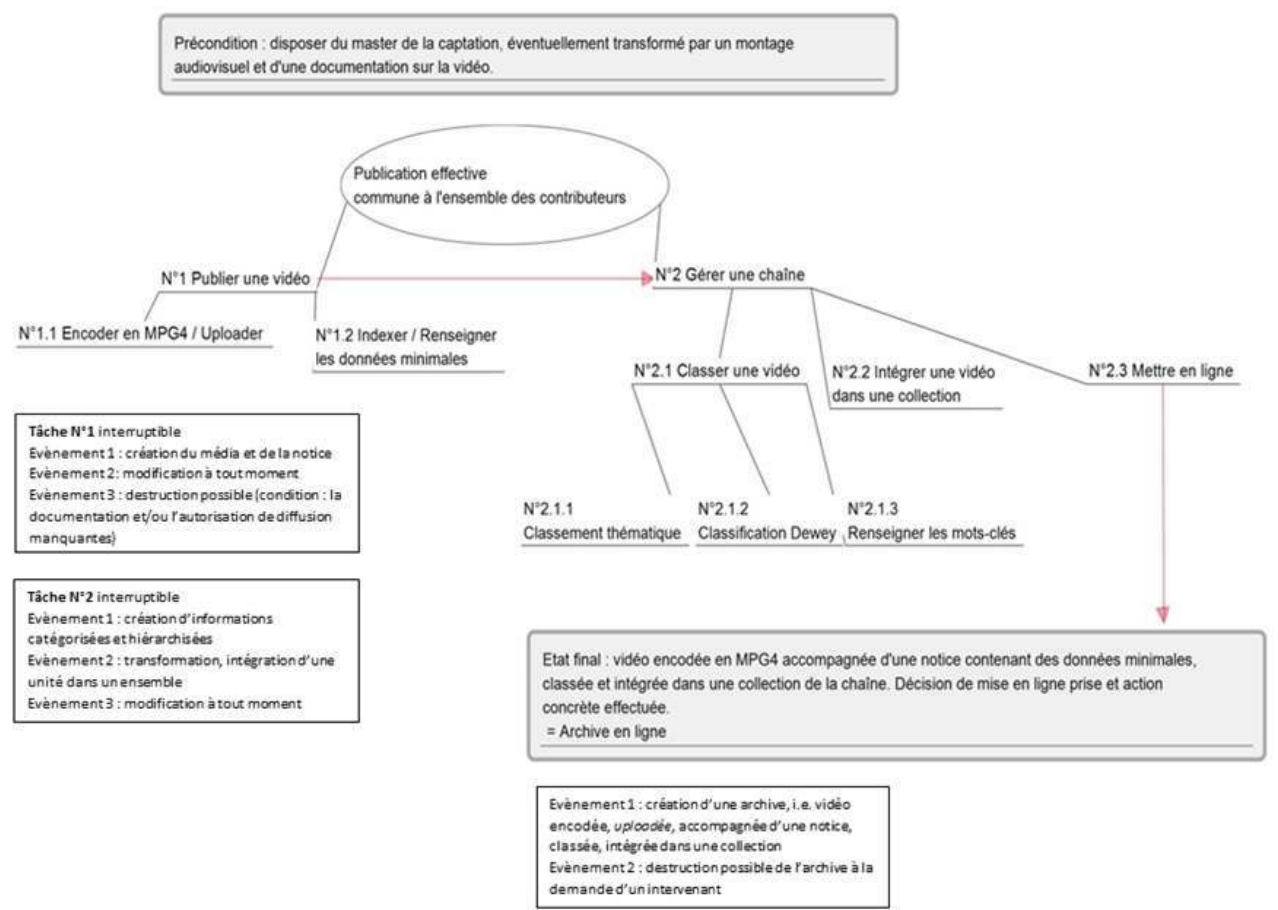

\section{Annexe 3 - Présentation des interfaces de Canal-U}


Figure 1 : Page d'accueil du dispositif. Figure 1 : Home page

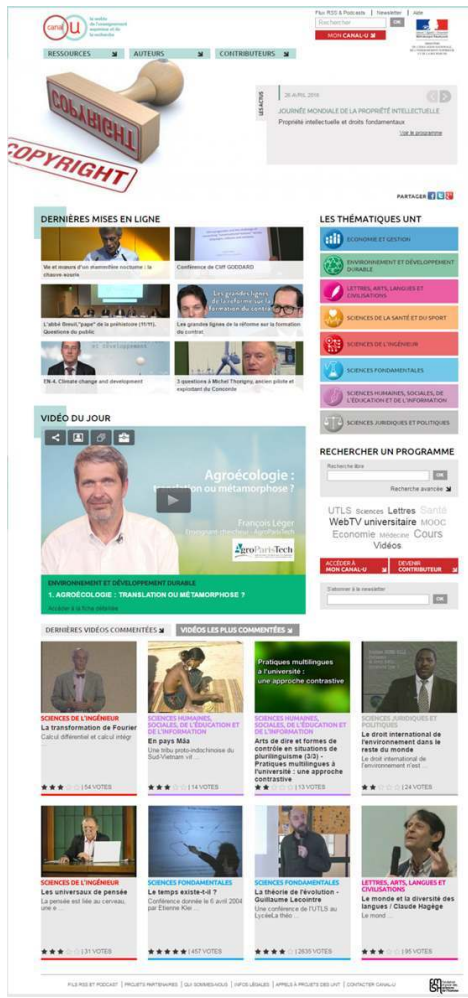

Figure 2 : Moteur de recherche avancée. Figure 2 : Advanced search engine

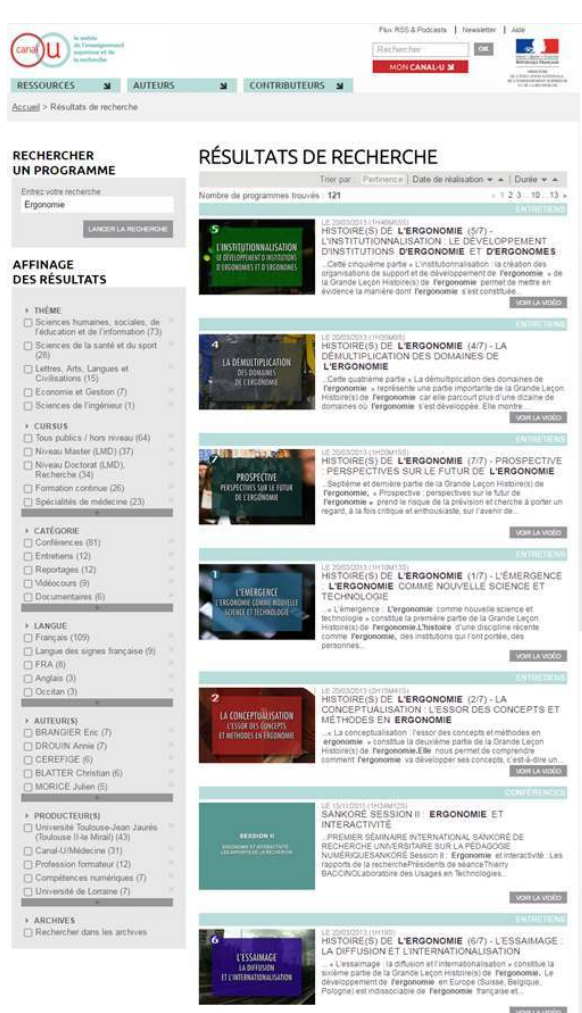


Figure 3 : Exemple de page de présentation des collections d'une chaîne. Figure 3 : Example of page presenting a channel's collections

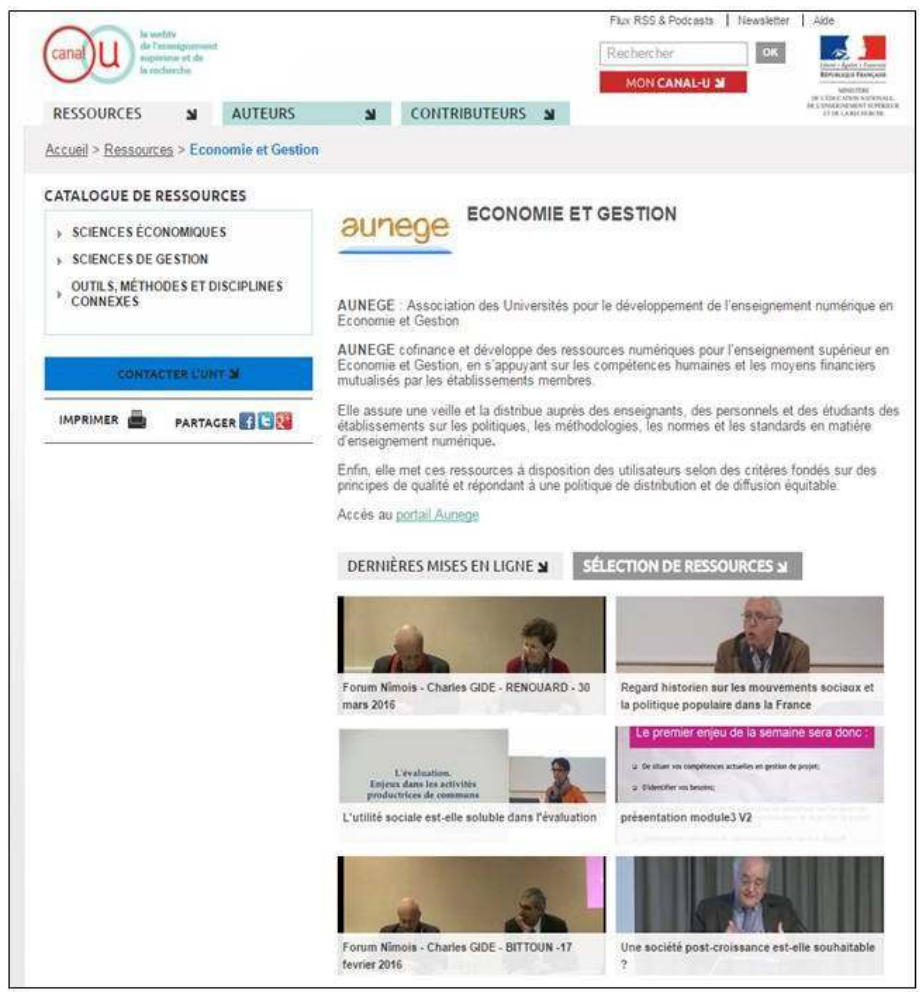

Figure 4 : Exemple d'interface de présentation et de lecture d'une vidéo. Figure 4 : Example of a video presentation and playback interface

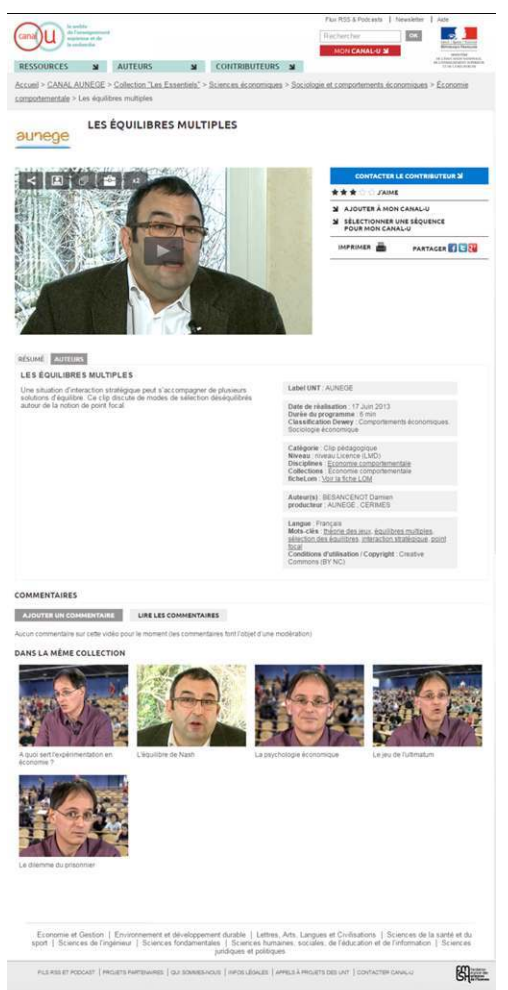




\section{Annexe 4 - Graphes de la tâche de recherche de vidéo}

Graphe de la tâche a priori de recherche de vidéo

La formalisation de la tâche a priori reflète l'activité de recherche de vidéo telle que la propose le système.

Graphe 8 : Tâches a priori de recherche de vidéo. Graph 8 : A priori video search tasks

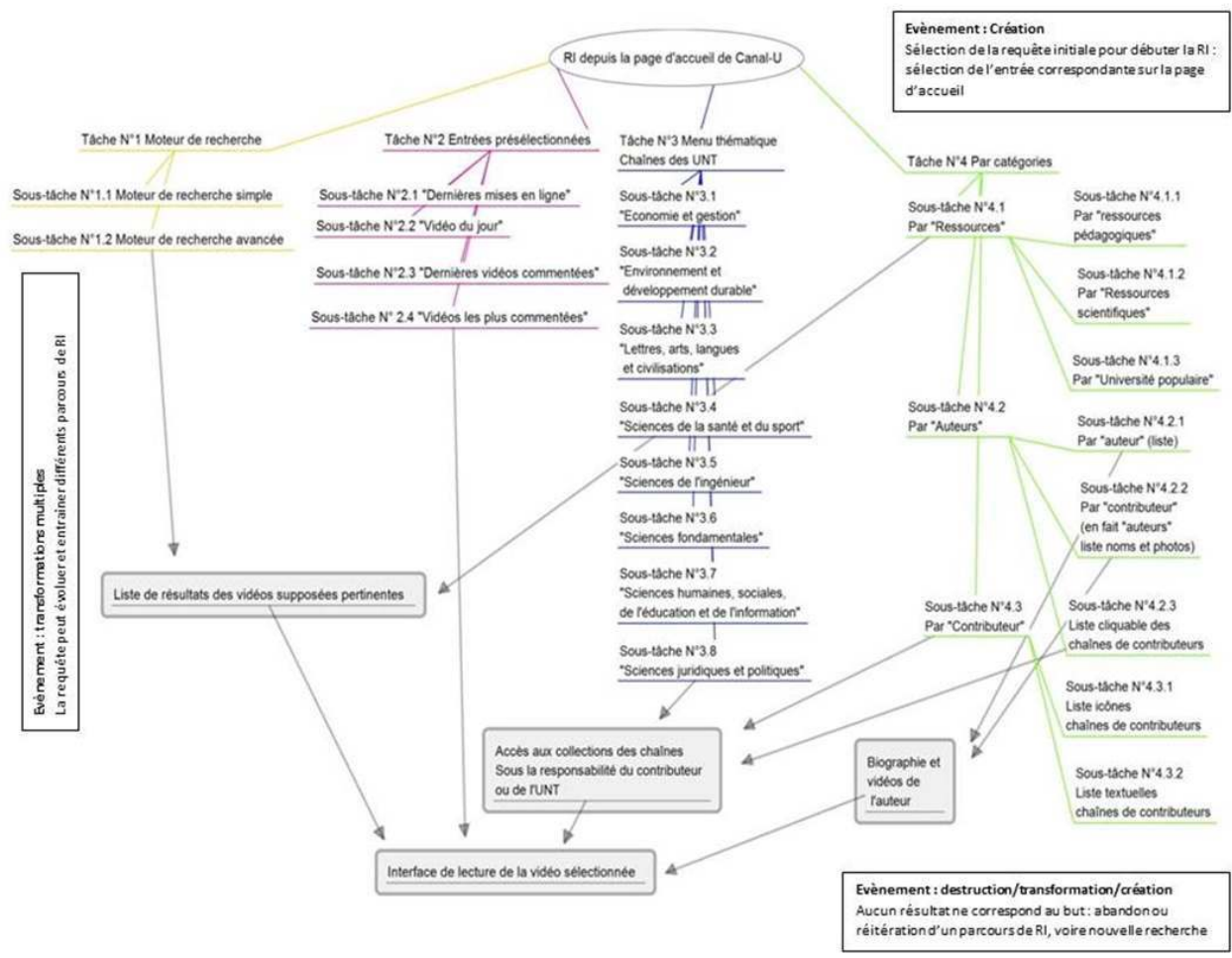

Graphe de la tâche effective commune de recherche de vidéo

Le graphe ci-dessous représente les tâches effectuées par l'ensemble des visiteurs interrogés. 
Graphe de la tâche effective commune de RI

Sous-tâche $N^{\circ} 1 . a$

Sélectionner des résultats dans la liste du moteur de recherche

Etat final: variable, la vidéo est visionnée immédiatement ou en différée, parfois transmise à un tiers.

\section{NOTES}

1. Téléchargement de fichiers audiovisuels pour une consultation hors-ligne.

2. Le TREC est un programme basé sur des workshops. Il fournit une infrastructure à une communauté de chercheurs leur permettant d'évaluer à grande échelle les méthodologies de recherche et le transfert de technologies.

3. TREC Video Retrieval Evaluation est centré sur l'analyse et la récupération de données basées sur le contenu des vidéos. Les principales publications concernent les algorithmes et l'automatisation.

4. http : //www-nlpir.nist.gov/projects/tv2017/

5. Le «modelage de l'environnement" correspond à une traduction libre de notre part de la terminologie de Pirolli et Card, «Enrichment activity ». L'expression choisie se justifie par une reconstruction de l'espace de recherche qui repose sur la sélection d'accès et de signaux conformes à la représentation que l'usager se fait de sa recherche.

6. «Parfum » est une traduction fidèle de la terminologie employée par Pirolli et Card, «Scent ». La théorie, basée sur une adaptation de la recherche de nourriture par les animaux, évoque ainsi l'odeur qu'ils poursuivent lorsqu'ils recherchent une proie.

7. Françoise Thibault, Dir., (2016), Rapport annuel de Canal-U pour le ministère de l'Enseignement supérieur et de la recherche, Fondation de la Maison des Sciences de l'Homme de Paris.

8. Ibid.

9. Il s'agit d'analyser le texte retranscrit en repérant les adverbes de temps (« en même temps que ", " avant ", «toujours », etc.), de liaison, de logique, les conjonctions de coordination, de subordination, etc.

10. Les professionnels de la documentation lui préfèrent souvent, dans le cadre de leur activité, celui de « chapitrage». 
11. Les visiteurs étrangers étaient francophones ou dominaient parfaitement le français.

12. L'ensemble des usagers possédait une excellente connaissance du dispositif et était en mesure de verbaliser leur parcours de navigation en décrivant avec précision les interfaces et les fonctionnalités utilisées. Aucun support n'a donc été utilisé pour les entretiens, ni en présentiel, ni à distance. Les conditions techniques étaient bonnes et les usagers, familiers de cette technologie. Nous avons donc considéré qu'il n'y avait pas de différences significatives sur ce point dans les deux conditions d'entretiens; l'utilisation de cette modalité étant d'autre part reconnue pour la collecte de données qualitatives (Sullivan, 2012).

13. Bien que se trouvant sur la notice, le résumé et les mots-clés appartiennent à la catégorie des substituts textuels puisqu'ils apportent une représentation compacte du contenu.

14. Ce but est décrit à partir du mode opératoire de l'enseignant en FLE.

15. Cette description est basée sur les déclarations de l'usager sans emploi avec un profil hors enseignement supérieur et recherche qui fréquente uniquement le dispositif pour se cultiver. Cependant les données relevées chez les interviewés qui poursuivent un but primaire autre, mais pour qui « se cultiver » représente un but secondaire, confirment les éléments décrits.

\section{RÉSUMÉS}

La publication et la recherche de vidéos sont deux activités humaines devenues très courantes ces dernières années et pourtant très peu étudiées. Ces deux activités complémentaires sont étudiées ici à travers les usages déclarés d'un dispositif académique en ligne. Sept responsables de la publication et huit utilisateurs du dispositif ont été interviewés sur leur activité, notamment sur leur but et la façon dont ils l'atteignent. Les résultats montrent que la réalisation de la tâche de publication, dans ses aspects techniques, éditoriaux, documentaires et relatifs à l'organisation des connaissances a un impact sur la tâche de consultation de l'usager à travers la compréhension du fonds et de l'organisation des connaissances, la localisation et l'identification d'une ressource, ainsi que les services favorisant le visionnage d'une vidéo. Les résultats mettent en évidence la présence de différentes catégories de buts de consultation et leurs modes opératoires. La discussion souligne les aspects sur lesquels l'intervention ergonomique dans ce type d'environnements académiques audiovisuels devrait porter.

Publishing and searching for videos online are two common human activities that remain underinvestigated today. We investigated these two related activities through interviews with users of an online academic audiovisual database. Seven publication managers and eight users were asked to explain their goals and strategies for publishing/searching for videos in the database. The results show that the way publication managers represent the publication task, in its technical, editorial, documentary and knowledge organization aspects, has an impact on the users' searching task, and more specifically on how they understand the collection and organization of knowledge, the location and identification of a resource, as well as the services supporting video viewing. These results suggest that different categories of consultation goals and modus operandi exist. The discussion examines the aspects that should be considered in the ergonomic design of this type of academic audiovisual database. 
INDEX

Keywords : information seeking, video browsing, video retrieval, audiovisual publication, academic

Mots-clés : recherche d'information, video browsing, video retrieval publication audiovisuelle, consultation de vidéos

\section{AUTEURS}

\section{EMMANUELLE PAPINOT}

Laboratoire Cognition, Langues, Langage et Ergonomie Toulouse II Maison de la Recherche 5, allées Antonio-Machado

31058 Toulouse Cedex 9

emmanuelle.papinot@msh-paris.fr

\section{ANDRÉ TRICOT}

Laboratoire Cognition, Langues, Langage et Ergonomie Toulouse II

Maison de la Recherche 5, allées Antonio-Machado

31058 Toulouse Cedex 9

andre.tricot@univ-tlse2.fr

\section{MÔNICA MACEDO-ROUET}

Experice Paris 8

2 rue de la Liberté

93526 Saint-Denis

monica.goncalves-macedo@univ-paris8.fr 